\title{
Goldstini Can Give the Higgs a Boost
}

\author{
Jesse Thaler and Zoe Thomas \\ Center for Theoretical Physics, Massachusetts Institute of Technology, \\ Cambridge, MA 02139, USA \\ E-mail: jthaler@jthaler.net, ztt@mit.edu
}

\begin{abstract}
Supersymmetric collider phenomenology depends crucially on whether the lightest observable-sector supersymmetric particle (LOSP) decays, and if so, what the LOSP decay products are. For instance, in SUSY models where the gravitino is lighter than the LOSP, the LOSP decays to its superpartner and a longitudinal gravitino via supercurrent couplings. In this paper, we show that LOSP decays can be substantially modified when there are multiple sectors that break supersymmetry, where in addition to the gravitino there are light uneaten goldstini. As a particularly striking example, a bino-like LOSP can have a near $100 \%$ branching fraction to a higgs boson and an uneaten goldstino, even if the LOSP has negligible higgsino fraction. This occurs because the uneaten goldstino is unconstrained by the supercurrent, allowing additional operators to mediate LOSP decay. These operators can be enhanced in the presence of a $U(1)_{R}$ symmetry, leading to copious boosted higgs production in SUSY cascade decays.
\end{abstract}




\section{Contents}

1. Introduction 2

2. Counterintuitive LOSP Decays 3

2.1 A Conventional Goldstino 4

2.2 Additional Operators? 5

2.3 Goldstini and $R$ Symmetries $\quad 7$

3. Goldstino and Gravitino Couplings $\quad 9$

3.1 The General Framework 9

3.2 The Decoupling and $R$-symmetric Limit 11

4. Higgsino Decoupling Limit Effective Field Theory 11

4.1 Leading $R$-symmetric Operators 11

4.2 Decay to Higgs Bosons 13

$\begin{array}{lll}4.3 & \text { Decay to } Z \text { Bosons } & 14\end{array}$

4.4 Decay to Difermions 15

5. Comparisons to the Gravitino Case $\mathbf{1 5}$

$\begin{array}{ll}5.1 \text { Additional Operations for the Gravitino } & 15\end{array}$

$\begin{array}{lll}5.2 & \text { Miraculous Cancellations } & 16\end{array}$

$\begin{array}{ll}5.3 \text { Why Goldstini are Different } & 17\end{array}$

6. Branching Ratio Results 18

$\begin{array}{lll}\text { 6.1 Higgs and } Z \text { Boson Branching Ratios } & 18\end{array}$

6.2 Difermion Branching Ratio 21

6.3 The $R$-violating Regime 22

$\begin{array}{ll}\text { 7. Conclusion } & 23\end{array}$

A. Tree-Level Higgs Potential 24

B. R-Symmetry Violating Decays 25

C. All-Orders Tree-Level Calculation $\quad 27$ 


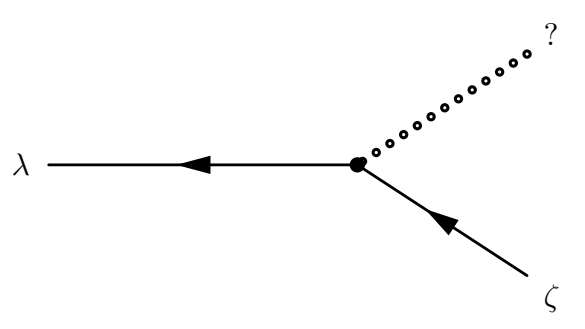

Figure 1: A generic LOSP decay. We will focus on the case where $\lambda$ is a bino-like LOSP, and $\zeta$ is a (pseudo-)goldstino from spontaneous SUSY breaking. Contrary to the naive expectation, $\lambda$ can decay dominantly to higgs bosons, even if $\lambda$ has negligible higgsino fraction.

\section{Introduction}

Supersymmetry (SUSY) is a well-motivated extension of the standard model (SM) with rich phenomenological implications for collider experiments like the LHC. Most SUSY theories consist of an "observable sector" coupled to one or more "hidden sectors." The observable sector contains the fields of the supersymmetric standard model (SSM), in particular the lightest observable-sector supersymmetric particle (LOSP). The hidden sectors are responsible for breaking SUSY and generating soft masses for SM superpartners, and may contain light states accessible to colliders.

A typical SUSY collider event involves production of two heavy SM superpartners which then undergo cascade decays to a pair of LOSPs. If there are hidden sector particles lighter than the LOSP, then the subsequent LOSP decays - if they occur inside the detector - can dramatically impact SUSY collider phenomenology. The most well-known example of a decaying LOSP is when the light hidden sector particle is a gravitino $[1,2,3,4]$. In that case, the LOSP decays to its superpartner and a longitudinal gravitino via interactions constrained by the conserved supercurrent and the goldstino equivalence theorem $[5,6,7,8]$. For example, a mostly bino LOSP will decay to a photon, $Z$, or-through its small higgsino fraction - a higgs boson.

In this paper, we will show how changes in the couplings between the observable and hidden sectors can have a dramatic impact on the decay modes of the LOSP, shown generically in Fig. 1. Our case study will be a nearly pure bino LOSP $\lambda$ with an order one branching fraction to higgs bosons, a very counterintuitive decay pattern from the point of view of the standard decay of a bino LOSP to a $\gamma / Z$ plus a longitudinal gravitino. In fact, in this example, the LOSP branching ratio to higgs bosons is enhanced with increasing higgsino mass $\mu$, approaching $100 \%$ in the small $\left(m_{\lambda} \tan \beta\right) / \mu$ limit. This is unlike the case of a higgsino LOSP, which generically has equal branching fractions to higgs and $Z$ bosons.

These novel bino LOSP decays are possible in the presence of multiple sectors which break supersymmetry, yielding a corresponding multiplicity of "goldstini" [9]. While the couplings of the true goldstino (eaten by the gravitino) are constrained by the supercurrent, the orthogonal uneaten goldstini can have different couplings from the naive expectation. The spectrum of goldstini exhibits a number of fascinating properties [9, 10, 11], and they may play a role in cosmology or dark matter $[12,13]$. Here, we will focus on properties of 


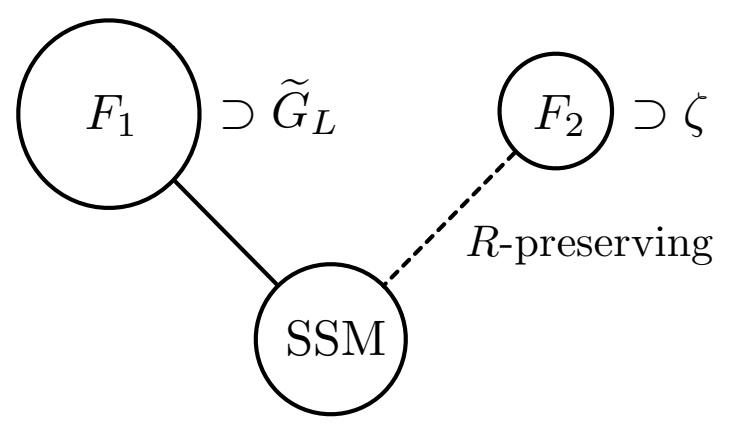

Figure 2: The $R$-symmetric setup that will be the focus of this paper. Here, sector 1 has a higher SUSY breaking scale than sector 2, i.e. $F_{1} \gg F_{2}$, so the LOSP preferentially decays to the pseudo-goldstino $\zeta$ coming mostly from sector 2 . Since sector 2 preserves an $R$ symmetry, the decay $\lambda \rightarrow \gamma / Z+\zeta$ is highly suppressed, and the mode $\lambda \rightarrow h^{0}+\zeta$ can dominate.

goldstini relevant for their collider phenomenology.

For our case study, we consider two sectors which break SUSY, both of which communicate to the SSM, but one of which preserves an $U(1)_{R}$ symmetry, as in Fig. 2. ${ }^{1}$ For the appropriate hierarchy of SUSY breaking scales, the LOSP will couple more strongly to the uneaten goldstino $\zeta$ than to the longitudinal gravitino $\widetilde{G}_{L}$. Since the uneaten goldstino $\zeta$ is charged under the $U(1)_{R}$ symmetry, the $R$-violating decay $\lambda \rightarrow \gamma / Z+\zeta$ is suppressed, letting the counterintuitive decay $\lambda \rightarrow h^{0}+\zeta$ dominate. $^{2}$ This fascinating result is demonstrated in Fig. 3.

In this way, goldstini can give the higgs a boost: a boost in production cross section since most LOSP decays yield a higgs boson; and a boost in kinematics since the higgses are produced with relatively large gamma factors in SUSY cascade decays. This example gives further motivation to identify boosted higgses using jet substructure techniques $[16,17,18]$. This example also motivates searches for other counter-intuitive LOSP decay patterns, where there is a mismatch between the identity of the LOSP and its decay products.

In the next section, we summarize and explain the main results of this paper. We then describe the framework of goldstini in Sec. 3, and derive the low energy effective goldstini interactions and resulting LOSP decay widths in Sec. 4. We explain in more detail why the goldstini case differs from the more familiar gravitino case in Sec. 5. Plots of the LOSP branching ratios appear in Sec. 6, and we conclude in Sec. 7. Various calculational details are left to the appendices.

\section{Counterintuitive LOSP Decays}

Throughout this paper, we will be considering the situation where a LOSP decays to a lighter neutral fermion as in Fig. 1, and we will assume the minimal SSM (MSSM)

\footnotetext{
${ }^{1}$ There have been recent studies where the entire SUSY breaking and SSM sectors preserve a $U(1)_{R}$ symmetry $[14,15]$.

${ }^{2}$ In Ref. [9], it was erroneously claimed that in the presence of an $R$ symmetry, the dominant decay is $\lambda \rightarrow \psi \bar{\psi}+\zeta$, where $\psi$ is a SM fermion. This paper corrects that error.
} 


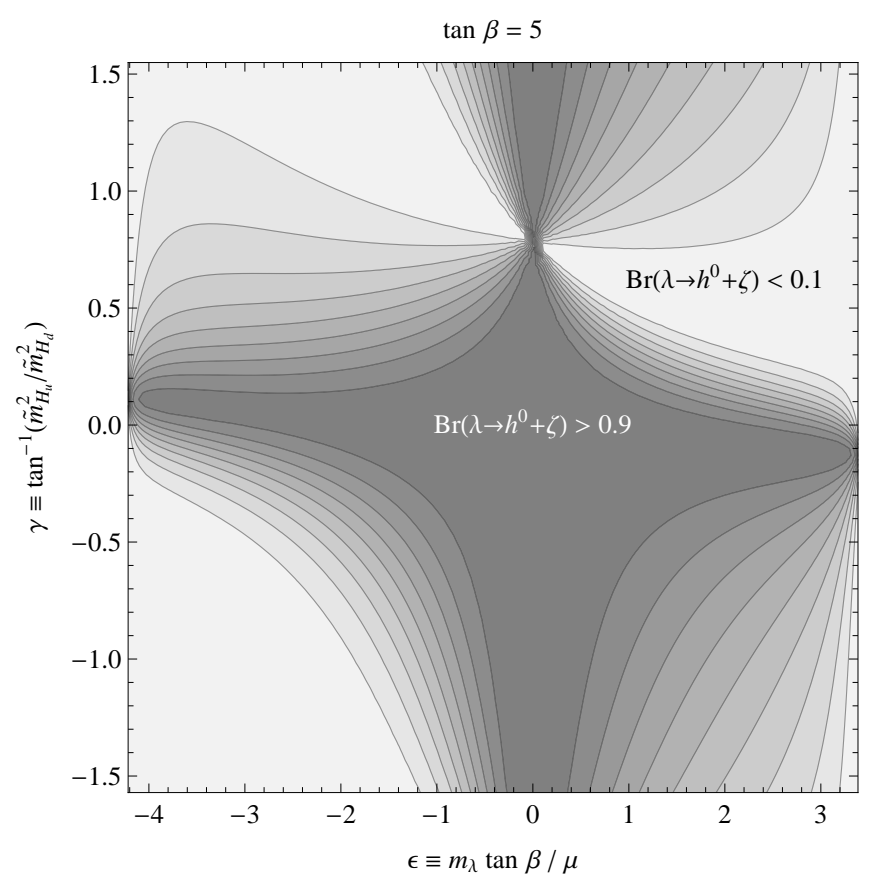

Figure 3: Branching ratio $\lambda \rightarrow h^{0}+\zeta$ for a bino LOSP in the $R$-symmetric setup from Fig. 2 . Throughout this parameter space, the remaining branching ratio is dominated by $\lambda \rightarrow Z+\zeta$. The expected mode $\lambda \rightarrow \gamma+\zeta$ is almost entirely absent. Shown is $\operatorname{Br}\left(\lambda \rightarrow h^{0} \zeta\right)$ as a function of $\epsilon \equiv m_{\lambda} \tan \beta / \mu$ and $\gamma \equiv \tan ^{-1}\left(\widetilde{m}_{H_{u}}^{2} / \widetilde{m}_{H_{d}}^{2}\right)$, fixing $\tan \beta=5, M_{1}=155 \mathrm{GeV}$, and $m_{h^{0}}=120 \mathrm{GeV}$. The plot terminates on the left and right side at the kinematic bound $m_{\lambda}<m_{h^{0}}$.

field content. The possible decay patterns of a LOSP are constrained by symmetries, at minimum conservation of SM charges. In the familiar case where the LOSP decays to its superpartner and a gravitino, there are further constraints imposed by conservation of the supercurrent. We will see that these constraints can be significantly relaxed in the presence of multiple SUSY breaking sectors.

\subsection{A Conventional Goldstino}

In the conventional setup with a single SUSY breaking sector and a light gravitino, the couplings of the helicity- $1 / 2$ components of the gravitino are linked via the goldstino equivalence theorem to the couplings of the goldstino $\widetilde{G}_{L}$. Supercurrent conservation implies that, at leading order in the inverse SUSY breaking scale $1 / F$, the goldstino couples only derivatively to observable sector fields via the supercurrent:

$$
\begin{aligned}
\mathcal{L}_{\text {eff }} & =i \widetilde{G}_{L}^{\dagger} \bar{\sigma}^{\mu} \partial_{\mu} \widetilde{G}_{L}+\frac{1}{F} \partial_{\mu} \widetilde{G}_{L} j^{\mu} \\
j^{\mu} & =\sigma^{\nu} \bar{\sigma}^{\mu} \psi_{i} D_{\nu} \phi^{* i}-\frac{1}{2 \sqrt{2}} \sigma^{\nu} \bar{\sigma}^{\rho} \sigma^{\mu} \lambda^{\dagger a} F_{\nu \rho}^{a}
\end{aligned}
$$

where we have elided terms that vanish on the goldstino equation of motion. Here, $\phi_{i}$ is a scalar and $\psi_{i}$ is its fermionic superpartner, and $F_{\mu \nu}^{a}$ is a gauge field strength with $\lambda^{a}$ its corresponding gaugino. In particular, the only possible LOSP decays are to its superpartner 

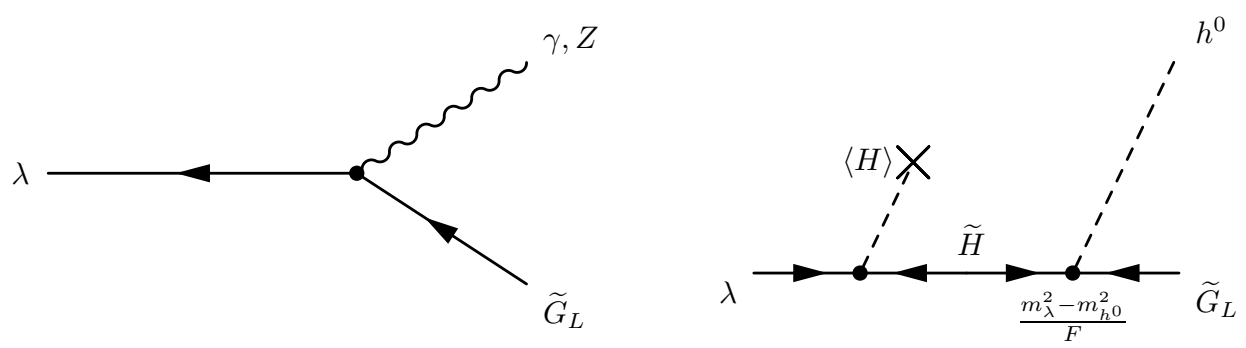

Figure 4: The standard decays of a bino-like LOSP to the longitudinal gravitino. They are primarily to a photon or $Z$ (left), though the bino may also decay to a higgs via its higgsino component (right). The derivatives in Eq. (2.2) yield the Yukawa coupling labeled here, proportional to the mass-squared difference of the on-shell bino and higgs. A cancellation between the two possible intermediate higgsinos means the propagator contributes a factor of $\mu^{-2}$ to the amplitude at leading order, leading to a very large suppression of this channel in the higgsino decoupling limit. Feynman diagrams throughout follow the conventions of Ref. [19].

and a gravitino. This implies, for example, that a pure right-handed stau LOSP $\tilde{\tau}_{R}$ can only decay to a gravitino and a right-helicity tau $\tau_{R}$, despite the fact that after electroweak symmetry breaking, there is no symmetry forbidding the decay to a left-helicity tau $\tau_{L}$.

For concreteness we will focus on a bino-like LOSP throughout this paper, though many of the following arguments hold with only minor modifications for a wino, as well. In that case, the supercurrent in Eq. (2.2) permits the decay $\lambda \rightarrow \gamma / Z+\widetilde{G}_{L}$ via the second term in the supercurrent. There is also a possible decay $\lambda \rightarrow h^{0}+\widetilde{G}_{L}$ where $h^{0}$ is the physical higgs boson, but since this occurs entirely through the higgsino fraction of the LOSP, it will be comparatively suppressed. ${ }^{3}$ Explicitly, to leading order in $m_{\lambda} / \mu$, the dominant LOSP partial widths are

$$
\begin{aligned}
\Gamma_{\gamma} & =\frac{m_{\lambda}^{5} \cos ^{2} \theta_{W}}{16 \pi F^{2}} \\
\Gamma_{Z} & =\frac{m_{\lambda}^{5} \sin ^{2} \theta_{W}}{16 \pi F^{2}}\left(1-\frac{M_{Z}^{2}}{m_{\lambda}^{2}}\right)^{4},
\end{aligned}
$$

where $m_{\lambda} \simeq M_{1}$ is the bino-like LOSP mass, and $\theta_{W}$ is the weak mixing angle. The subdominant width to higgs bosons is

$$
\Gamma_{h^{0}}=\frac{m_{\lambda}^{2} M_{Z}^{2}}{\mu^{4}} \frac{m_{\lambda}^{5} \sin ^{2} \theta_{W} \cos ^{2} 2 \beta}{32 \pi F^{2}}\left(1-\frac{m_{h^{0}}^{2}}{m_{\lambda}^{2}}\right)^{2},
$$

where $\tan \beta \equiv v_{u} / v_{d}$. Feynman diagrams for these standard decays are shown in Fig. 4 .

\subsection{Additional Operators?}

In the case of the true goldstino $\widetilde{G}_{L}$, its couplings are saturated by Eq. (2.2). But if the LOSP were to decay not to a true goldstino but to a generic neutral fermion $\zeta$, then

\footnotetext{
${ }^{3}$ See Ref. [20] for a recent discussion of more general neutralino decays.
} 

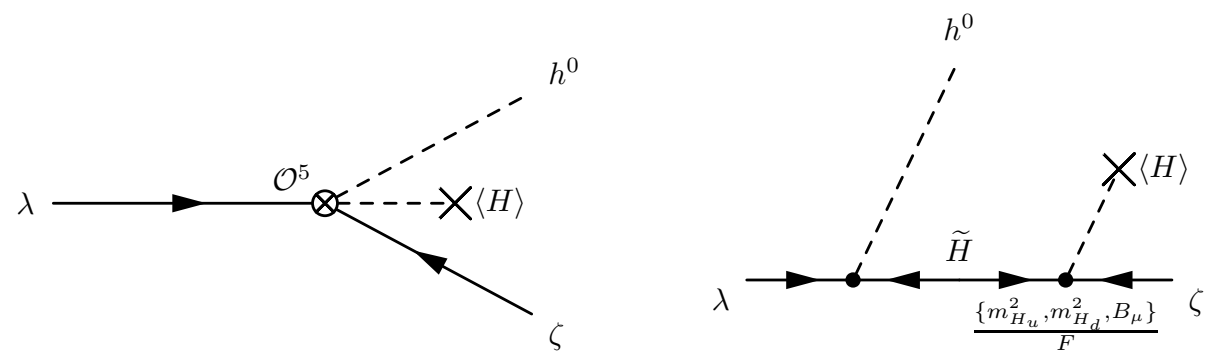

Figure 5: Additional diagrams which could contribute to LOSP decay. The dimension 5 operator (left) can be generated by integrating out an intermediate higgsino (right). There is also a diagram with $h^{0}$ and $\langle H\rangle$ reversed. However, if $\zeta$ is a longitudinal gravitino $\widetilde{G}_{L}$, then the width $\Gamma\left(\lambda \rightarrow h^{0}+\zeta\right)$ vanishes in the higgsino decoupling limit.

there are many more operators that might mediate LOSP decay instead. For example, the dimension 5 operator

$$
\mathcal{O}_{R}^{5}=C_{R}^{5} \frac{\mu}{F} \lambda \zeta\left(H_{u} \cdot H_{d}\right)^{*} \quad\left(\lambda \rightarrow h^{0}+\zeta\right)
$$

mediates the decay $\lambda \rightarrow h^{0}+\zeta$ after electroweak symmetry breaking. Here, the coefficient $\mu / F$ has been chosen with malice aforethought, as this will turn out to be the approximate scaling behavior for the eaten goldstino. The subscript $R$ indicates that this operator will preserve a $U(1)_{R}$ symmetry once we identify $\zeta$ with an uneaten goldstino of $R$-charge 1 .

There are also additional operators at dimension 5 which violate this $U(1)_{R}$ symmetry,

$$
\begin{aligned}
\mathcal{O}_{\not R, u \cdot d}^{5} & =C_{\not R, u}^{5} \frac{\mu}{F} \lambda \zeta\left(H_{u} \cdot H_{d}\right), \\
\mathcal{O}_{\not R, u}^{5} & =C_{\not R, u}^{5} \frac{\mu}{F} \lambda \zeta H_{u}^{\dagger} H_{u}, \\
\mathcal{O}_{\not R, d}^{5} & =C_{\not R, d}^{5} \frac{\mu}{F} \lambda \zeta H_{d}^{\dagger} H_{d} .
\end{aligned}
$$

Considering these $\mathcal{O}^{5}$ operators together, the partial width for the decay $\lambda \rightarrow h^{0}+\zeta$ is

$$
\Gamma_{h^{0}}=\left(C_{\text {net }}^{5}\right)^{2} \frac{\mu^{2} M_{Z}^{2}}{m_{\lambda}^{4}} \frac{m_{\lambda}^{5} \sin ^{2} \theta_{W}}{32 \pi F^{2}}\left(1-\frac{m_{h^{0}}^{2}}{m_{\lambda}^{2}}\right)^{2} .
$$

Here, we have defined

$$
C_{\mathrm{net}}^{5}=\frac{\sqrt{2}}{g^{\prime}}\left(\left(C_{R}^{5}+C_{\not h, u \cdot d}^{5}\right) \cos (\alpha+\beta)-2 C_{\not h, u}^{5} \sin \beta \cos \alpha+2 C_{\not R, d}^{5} \cos \beta \sin \alpha\right),
$$

with $\alpha$ being the physical higgs mixing angle. Thus, if somehow the $\mathcal{O}^{5}$ operators were dominant over operators like those in Eq. (2.2), then the decay of a pure bino LOSP to a higgs would dominate over the decay to a $\gamma / Z$. Note that the $\mathcal{O}^{5}$ operators only mediate a decay to one or more higgs bosons, and not to a longitudinal $Z$, due to the gauge invariance of the scalar portion of the operators.

Now, in the conventional goldstino case, there is a sense in which the $\mathcal{O}^{5}$ operators are indeed generated after integrating out the higgsino as in Fig. 5. This occurs not in the 
derivatively-coupled basis, but rather in the non-linear goldstino basis described in Sec. 3 . The pertinent combination of Wilson coefficients attains the value

$$
C_{\mathrm{net}}^{5}=\frac{\left(m_{H_{u}}^{2}-m_{H_{d}}^{2}\right) \sin 2 \beta+2 B_{\mu} \cos 2 \beta}{\mu^{2}}+\mathcal{O}\left(\frac{m_{\lambda}}{\mu}\right)
$$

which MSSM aficionados will recognize as being zero for the tree-level higgs potential in the decoupling limit $|\mu| \gg M_{Z}$ - the same limit in which it was legitimate to integrate out the higgsinos in the first place (see App. A for an explanation of this cancellation). This is as it must be; the physical predictions in this field basis must agree with those of the basis corresponding to the supercurrent picture of Eq. (2.2), in which the decay rate to higgs bosons is highly suppressed.

However, because $C_{\text {net }}^{5}=0$ arises only because of a delicate cancellation in the true goldstino case, any deviation will give rise to additional LOSP decays beyond the supercurrent prediction. In particular, if there are multiple sectors that break SUSY [9], each of which contributes only partially to the SSM soft masses, then the couplings of the uneaten goldstini cannot be determined by supercurrent considerations. ${ }^{4}$ In general, the goldstini will have very different couplings from the gravitino; concretely, the goldstini need not be derivatively coupled to observable-sector particles. For a generic uneaten goldstino

$$
C_{\text {net }}^{5}=\frac{\left(\widetilde{m}_{H_{u}}^{2}-\widetilde{m}_{H_{d}}^{2}\right) \sin 2 \beta+2 \widetilde{B}_{\mu} \cos 2 \beta}{\mu^{2}}+\mathcal{O}\left(\frac{m_{\lambda}}{\mu}\right),
$$

where the tildes indicate the linear combination, appropriate to the given goldstino, of contributions from the SUSY-breaking sectors to the corresponding soft mass. These parameters need not cancel and thus a pure bino LOSP can exhibit the counterintuitive decay to a higgs boson and an uneaten goldstino.

\subsection{Goldstini and $R$ Symmetries}

The differences between LOSP decays to an eaten goldstino versus an uneaten goldstino become especially striking in the presence of a $U(1)_{R}$ symmetry, and they will be the main example in this paper. Consider the case of two SUSY breaking sectors as in Fig. 2 where the uneaten goldstino is associated with a sector 2 that preserves an $R$-symmetry. As we will argue in Sec. 3, if the scale of SUSY breaking in sector 1 is much higher than in sector 2, i.e. $F_{1} \gg F_{2}$, then we can ignore the standard LOSP decay to a gravitino, since it will be overwhelmed by the LOSP decay to the uneaten goldstino from sector 2 .

The gaugino soft mass terms violate the $R$-symmetry, so a bino LOSP cannot undergo the associated decay to a $\gamma / Z$ and the uneaten goldstino $\zeta$. Instead, it must (at tree level) decay to the uneaten goldstino via a virtual higgsino or sfermion as in Figs. 5 and 6 , producing a higgs $h^{0}$, an arbitarily-polarized $Z$, or two SM fermions $\psi \bar{\psi}$ in the process.

To understand this effect more clearly, note there are only a limited number of $R$ symmetric operators that can mediate the decay of a bino LOSP to an uneaten goldstino and standard model particles once the higgsinos and sfermions are integrated out. At

\footnotetext{
${ }^{4}$ This fact was recently exploited in Ref. [13] to arrange for goldstini dark matter with leptophilic decays.
} 

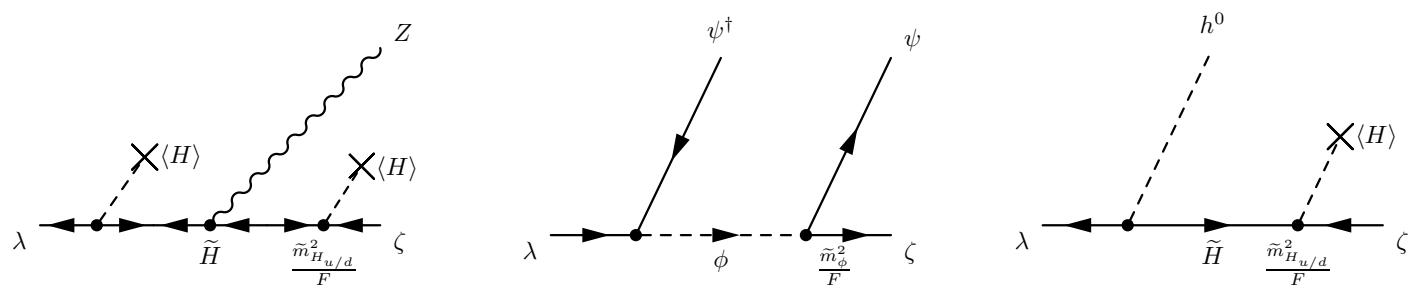

Figure 6: Representative diagrams contributing to the dimension 6 operators. After integrating out the intermediate higgsinos or sfermions, these diagrams mediate LOSP decays to $Z$ bosons and SM difermions, as well as generating additional LOSP decays to $h^{0}$.

dimension 5, only $\mathcal{O}_{R}^{5}$ respects the $R$-symmetry; the other $\mathcal{O}^{5}$ operators are associated with the $R$-symmetry-violating $B_{\mu}$ term. At dimension 6 , we will show that the only operators consistent with gauge symmetries, $R$-parity, and our imposed $R$-symmetry are

$$
\begin{array}{rlrl}
\mathcal{O}_{\Phi, 1}^{6} & =\frac{C_{\Phi, 1}^{6} i \zeta^{\dagger} \bar{\sigma}^{\mu} \lambda \Phi^{\dagger} D_{\mu} \Phi}{F} & \left(\lambda \rightarrow h^{0} / Z+\zeta\right), \\
\mathcal{O}_{\Phi, 2}^{6}=\frac{C_{\Phi, 2}^{6}}{F} i \zeta^{\dagger} \bar{\sigma}^{\mu} \lambda\left(D_{\mu} \Phi^{\dagger}\right) \Phi & \left(\lambda \rightarrow h^{0} / Z+\zeta\right), \\
\mathcal{O}_{\psi}^{6}=\frac{C_{\psi}^{6}}{F}\left(\zeta^{\dagger} \psi^{\dagger}\right)(\psi \lambda) & (\lambda \rightarrow \psi \bar{\psi}+\zeta),
\end{array}
$$

where $\Phi$ stands for either $H_{u}$ or $H_{d}, \psi$ is an SM fermion, and we have indicated in parentheses the corresponding LOSP decay mode. The values of the Wilson coefficients $C^{6}$ are omitted here for clarity; they are given explicitly in Eq. (4.5). Despite the fact that we have integrated out a higgsino/sfermion, these operators are not suppressed by the higgsino/sfermion mass as there is a cancellation between the propagator of the virtual heavy particle and its coupling to the goldstino. We will explain this fact in more detail in Sec. 4; it is sufficient to note for now that the $\mathcal{O}_{\Phi}^{6}$ are suppressed by a power of $\mu$ relative to $\mathcal{O}_{R}^{5}$.

The relative importance of $\mathcal{O}_{R}^{5}, \mathcal{O}_{\Phi, i}^{6}$, and $\mathcal{O}_{\psi}^{6}$ for LOSP decays depend sensitively on the SSM parameters. In general, the three-body decay $\lambda \rightarrow \psi \bar{\psi}+\zeta$ is subdominant to the two-body decays $\lambda \rightarrow h^{0} / Z+\zeta$. As mentioned already, $\mathcal{O}_{R}^{5}$ only mediates a decay to higgs bosons, not to longitudinal $Z$ bosons, whereas $\mathcal{O}_{\Phi, i}^{6}$ can yield either, or even a transverse $Z$. One might naively expect $\mathcal{O}_{R}^{5}$ to dominate over $\mathcal{O}_{\Phi, i}^{6}$, since the dimension 6 operator has a decay amplitude suppressed by $m_{\lambda} / \mu$. However, $\mathcal{O}_{R}^{5}$ contains $H_{u} \cdot H_{d}$ which involves an additional $1 / \tan \beta$ suppression in the large $\tan \beta$ limit, while the operators $\mathcal{O}_{H_{u}, i}^{6}$ have no such suppression. Thus, the dimension 6 decays are only suppressed by

$$
\epsilon \equiv \frac{m_{\lambda} \tan \beta}{\mu}
$$

compared to the dimension 5 decays, which may not even be a suppression at large $\tan \beta$.

In Fig. 3, we showed the LOSP branching ratios as a function of both $\epsilon$ and the most important other free parameter in the theory

$$
\tan \gamma \equiv \frac{\widetilde{m}_{H_{u}}^{2}}{\widetilde{m}_{H_{d}}^{2}},
$$


which is the ratio of the contributions to $m_{H_{u}}^{2}$ and $m_{H_{d}}^{2}$ from the sector containing the uneaten goldstino. For special values of $\gamma$, the decay mode $\lambda \rightarrow Z+\zeta$ can either be completely suppressed or enhanced relative to $\lambda \rightarrow h^{0}+\zeta$ due to cancellations. Our main interest will be in the higgsino decoupling limit with small $\epsilon$, where the higgs mode generically dominates.

Thus, in the presence of a $R$-symmetry, the LOSP decay to an uneaten goldstino gives a boost to higgs boson production, even if (and especially if) the LOSP has a negligible higgsino fraction. Moreover, the decays to the uneaten goldstino, whether featuring a higgs boson or not, will completely dominate over any decays to the gravitino if there is an appropriate hierarchy between the two SUSY-breaking scales, as we will describe in the next section.

\section{Goldstino and Gravitino Couplings}

Having understood the possibility of enhanced $\lambda \rightarrow h^{0}+\zeta$ decays from an operator perspective, the remainder of this paper will show how precisely this works in the explicit example of multiple SUSY breaking sectors.

\subsection{The General Framework}

As in Ref. [9], we consider two sequestered sectors, each of which spontaneously breaks SUSY. Each sector has an associated goldstino ( $\eta_{1}$ and $\eta_{2}$, respectively), and we characterize the size of SUSY breaking via the goldstino decay constants ( $F_{1}$ and $F_{2}$, respectively). Each SUSY breaking sector can be parametrized in terms of a non-linear goldstino multiplet $[21,9]$

$$
X_{i}=\frac{\eta_{i}^{2}}{2 F_{i}}+\sqrt{2} \theta \eta_{i}+\theta^{2} F_{i}
$$

for $i=1,2$. We define the quantities

$$
F \equiv \sqrt{F_{1}^{2}+F_{2}^{2}}, \quad \tan \theta \equiv \frac{F_{2}}{F_{1}},
$$

and we take $\tan \theta \leq 1\left(F_{1} \geq F_{2}\right)$ without loss of generality.

The combination $\widetilde{G}_{L}=\sin \theta \eta_{1}+\cos \theta \eta_{2}$ is eaten by the gravitino to become its longitudinal components via the super-higgs mechanism, but the orthogonal goldstino $\zeta=\cos \theta \eta_{1}-\sin \theta \eta_{2}$ remains uneaten and will be the focus of our study. For simplicity, we will work in the $M_{\mathrm{Pl}} \rightarrow \infty$ limit where the uneaten goldstino remains massless, though in general $\zeta$ will get a mass proportional to $m_{3 / 2}$ via SUGRA effects, in particular $m_{\zeta}=2 m_{3 / 2}$ in the minimal goldstini scenario [9]. In addition, variations in the SUSYbreaking dynamics [10] or induced couplings between the two sectors $[9,11]$ can modify the mass term for $\zeta .^{5}$

\footnotetext{
${ }^{5}$ At minimum, one expects loops of SM fields to generate $m_{\zeta} \simeq m_{\text {soft }} /\left(16 \pi^{2}\right)^{n}$ [9], where $n$ depends on the number of loops necessary to effectively connect sectors 1 and 2 and transmit the needed $U(1)_{R}$ breaking. The uneaten goldstino will also obtain a tree-level mass due to mixing with the neutralinos, but this is of order $1 / F^{2}$ and is comparatively negligible.
} 
Supersymmetry breaking is communicated from the two hidden sectors to the visible sector by means of a non-trivial Kähler potential and gauge kinetic function (presumably coming from integrating out heavy messenger fields). Some representative terms contributing to the SSM soft masses are ${ }^{6}$

$$
\begin{aligned}
K & =\Phi^{\dagger} \Phi \sum_{i} \frac{m_{\phi, i}^{2}}{F_{i}} X_{i}^{\dagger} X_{i} \\
f_{a b} & =\frac{1}{g_{a}^{2}} \delta_{a b}\left(1+\sum_{i} \frac{2 M_{a, i}}{F_{i}} X_{i}\right),
\end{aligned}
$$

where $i=1,2$, and $\Phi$ stands for a general SSM multiplet. These yield the following terms in the lagrangian up to order $1 / F[22]$ :

$$
\begin{aligned}
\mathcal{L}= & -\sum_{i} m_{\phi, i}^{2} \phi^{*} \phi+\sum_{i} \frac{m_{\phi, i}^{2}}{F_{i}} \eta_{i} \psi \phi^{*} \\
& -\frac{1}{2} \sum_{i} M_{a, i} \lambda^{a} \lambda^{a}-\sum_{i} \frac{i M_{a, i}}{\sqrt{2} F_{i}} \eta_{i} \sigma^{\mu \nu} \lambda^{a} F_{\mu \nu}^{a}+\sum_{i} \frac{M_{a, i}}{\sqrt{2} F_{i}} \eta_{i} \lambda^{a} D^{a} .
\end{aligned}
$$

Thus, the parameter $m_{\phi, i}^{2}\left(M_{a, i}\right)$ is the contribution to the SUSY-breaking scalar (gaugino) mass from each respective sector. Note that they are intrinsically related to the coupling of the SSM fields to the goldstini.

Rotating to the $\widetilde{G}_{L}-\zeta$ basis yields similar interaction terms for the eaten goldstino $\widetilde{G}_{L}$ and the uneaten goldstino $\zeta$,

$$
\begin{aligned}
\mathcal{L}_{\widetilde{G}_{L}} & =\frac{m_{\phi}^{2}}{F} \widetilde{G}_{L} \psi \phi^{*}-\frac{i M_{a}}{\sqrt{2} F} \widetilde{G}_{L} \sigma^{\mu \nu} \lambda^{a} F_{\mu \nu}^{a}+\frac{M_{a}}{\sqrt{2} F} \widetilde{G}_{L} \lambda^{a} D^{a}, \\
\mathcal{L}_{\zeta} & =\frac{\widetilde{m}_{\phi}^{2}}{F} \zeta \psi \phi^{*}-\frac{i \widetilde{M}_{a}}{\sqrt{2} F} \zeta \sigma^{\mu \nu} \lambda^{a} F_{\mu \nu}^{a}+\frac{\widetilde{M}_{a}}{\sqrt{2} F} \zeta \lambda^{a} D^{a},
\end{aligned}
$$

where the untilded and tilded mass parameters associated with gauginos denote

$$
\begin{aligned}
& M_{a}=M_{a, 1}+M_{a, 2}, \\
& \widetilde{M}_{a}=M_{a, 2} \cot \theta-M_{a, 1} \tan \theta,
\end{aligned}
$$

with the analogous notation for the scalar mass-squared parameters. Throughout, we will work in the limit $\cot \theta \gg 1$, for which we can take

$$
\frac{\widetilde{M}_{a}}{F}=\frac{M_{a, 2}}{F_{2}}, \quad \frac{\widetilde{m}_{\phi}^{2}}{F}=\frac{m_{\phi, 2}^{2}}{F_{2}} .
$$

In this limit, as long as any of the $M_{a, 2}$ or $m_{\phi, 2}^{2}$ are at least on the order of the weak scale, LOSP decays to gravitinos are very suppressed and can be ignored for collider purposes. We see that as predicted via the supercurrent, the true goldstino $\widetilde{G}_{L}$ couples to SSM fields in proportion to the physical soft masses. In contrast, $\zeta$ couples via the tilded mass parameters which in the $\cot \theta \gg 1$ limit are proportional just to the contribution of sector 2 to the SSM soft masses.

\footnotetext{
${ }^{6}$ We only give the Kähler potential for a single species of scalar; more general $A$ and $B$-terms involving multiple species can also be formed.
} 


\subsection{The Decoupling and $R$-symmetric Limit}

In this paper, we will focus on the higgsino decoupling and $R$-symmetric limits. That is, we will be considering the limit where $\mu$ is large compared to $m_{\lambda}$, and the limit where sector 2 preserves a $U(1)_{R}$ symmetry. There are a number of important features of this limit.

When the higgsinos are decoupled, the soft terms $m_{H_{u}}^{2}, m_{H_{d}}^{2}$, and $B_{\mu}$ must scale as $\mathcal{O}\left(\mu^{2}\right)$ in order to get successful electroweak symmetry breaking. ${ }^{7}$ We can see from the above lagrangian that the coupling of $\widetilde{G}_{L}$ to a higgsino and a higgs is proportional to these $\mathcal{O}\left(\mu^{2}\right)$ soft SUSY-breaking masses. The same is true for the couplings of $\zeta$ if we make the additional simplifying assumption that the tilded mass parameters scale in the same fashion, so long as this is not forbidden by a symmetry. With one noted exception in Sec. 6.2, however, our results do not depend on this assumption. From the diagrams in Fig. 5, one would naively expect the amplitudes for the decay of a bino LOSP to the physical higgs and either goldstino via a virtual higgsino to be of order $\mu$ and thus dominant over other decays to the same goldstino in the decoupling limit. As we will argue in Sec. 5.2, there is a cancellation in the $\widetilde{G}_{L}$ case which renders the decay $\lambda \rightarrow h^{0}+\widetilde{G}_{L}$ small, whereas for $\zeta$, the decay $\lambda \rightarrow h^{0}+\zeta$ can indeed dominate.

In the limit where sector 2 is $R$-symmetric, the contribution from sector 2 to SSM $A$-terms, $B$-terms, and gaugino masses is zero. Most relevant for our purposes, this implies that $\widetilde{B}_{\mu}$ and $\widetilde{M}_{1}$ are nearly zero. The absence of a $\widetilde{B}_{\mu}$ term implies that the cancellation in Eq. (2.12) seen for $\widetilde{G}_{L}$ cannot persist for the uneaten goldstino $\zeta$. The absence of a $\widetilde{M}_{1}$ term means that the LOSP decay to a $\gamma / Z$ and $\zeta$ is highly suppressed. ${ }^{8}$ Both of these facts imply a large $\lambda \rightarrow h^{0}+\zeta$ branching fraction. Depending on the relative importance of the dimension 5 or dimension 6 operators, the mode $\lambda \rightarrow Z+\zeta$ can be large as well.

\section{Higgsino Decoupling Limit Effective Field Theory}

Starting from the above goldstini framework, we can now systematically describe which operators contribute to bino LOSP decay in the higgsino decoupling and $R$-symmetric limits. We will then give the resulting decay rates for the three main decay modes: $\lambda \rightarrow$ $h^{0}+\zeta, \lambda \rightarrow Z+\zeta$, and $\lambda \rightarrow \psi \bar{\psi}+\zeta$.

\subsection{Leading $R$-symmetric Operators}

In the higgsino decoupling limit, it is convenient to organize the LOSP decay operators in terms of the small parameter $m_{\lambda} / \mu$. This may be accomplished practically by integrating out the heavy higgsino degrees of freedom, yielding an effective field theory with successively higher-dimension operators suppressed by additional powers of $\mu$. Away from the

\footnotetext{
${ }^{7}$ Strictly speaking, this is only true for the combinations $m_{H_{u}}^{2}$ and $m_{H_{d}}^{2}+B_{\mu} \tan \beta$ (working in the large $\tan \beta$ limit). However, if one simultaneously decouples the heavy higgs scalars in the same way, so that $m_{A^{0}}^{2}$ is of order $\mu^{2}$, then all three soft mass parameters scale as $\mu^{2}$ barring accidental cancellations. Our later results for the uneaten goldstino are robust against this assumption, since $m_{H_{u}}^{2}$ has the desired scaling properties regardless.

${ }^{8}$ In the alternative limit where sector 1 preserves an $R$-symmetry, one expects $\lambda \rightarrow \gamma / Z+\zeta$ to still be relevant, but that will not be the focus of this paper.
} 
decoupling limit, App. C describes how to calculate the LOSP branching fractions for arbitrary $\mu$. For simplicity, we will take $F_{1} \gg F_{2}$, in which case the couplings of the uneaten goldstino are completely determined by sector 2 .

Recall that in the MSSM, gauginos have $R$-charge 1 , higgs multiplets have $R$-charge 1 , and matter multiplets have $R$-charge $1 / 2$. For an $R$-symmetric SUSY breaking sector, the corresponding goldstino has $R$-charge 1 . Putting this together, at dimension 5 , there is only a single operator contributing to bino LOSP decay consistent with the symmetries of the theory (including the imposed $R$-symmetry):

$$
\mathcal{O}_{R}^{5}=C_{R}^{5} \frac{\mu}{F} \lambda \zeta\left(H_{u} \cdot H_{d}\right)^{*}
$$

This operator may mediate the decay of a bino LOSP to the uneaten goldstino and one or two physical higgs bosons $h^{0}{ }^{9}$

At dimension 6 , there are three sorts of additional operators: ${ }^{10}$

$$
\begin{aligned}
\mathcal{O}_{\Phi, 1}^{6} & =\frac{C_{\Phi, 1}^{6}}{F} i \zeta^{\dagger} \bar{\sigma}^{\mu} \lambda \Phi^{\dagger} D_{\mu} \Phi, \\
\mathcal{O}_{\Phi, 2}^{6} & =\frac{C_{\Phi, 2}^{6}}{F} i \zeta^{\dagger} \bar{\sigma}^{\mu} \lambda\left(D_{\mu} \Phi^{\dagger}\right) \Phi, \\
\mathcal{O}_{\psi}^{6} & =\frac{C_{\psi}^{6}}{F}\left(\zeta^{\dagger} \psi^{\dagger}\right)(\psi \lambda),
\end{aligned}
$$

where $\Phi$ stands for either $H_{u}$ or $H_{d}$, and $\psi$ is a standard model fermion. The dimension 6 operators $\mathcal{O}_{\Phi, i}^{6}$ may produce a $Z$ boson (longitudinal or otherwise) instead of or in addition to any higgs boson production. The dimension 6 operator $\mathcal{O}_{\psi}^{6}$ will produce a difermion pair instead. ${ }^{11}$ The effects of $\mathcal{O}_{\psi}^{6}$, but not the others, were considered in Ref. [9].

We have omitted two possible $R$-symmetric operators, $\partial^{\mu} \zeta \sigma^{\nu} \lambda^{\dagger} F_{\mu \nu}$ and $\partial^{\mu} \zeta \sigma^{\nu} \lambda^{\dagger} \widetilde{F}_{\mu \nu}$, which could mediate the decay of the bino to a photon or $Z$ and the goldstino. It is clear by examining the original lagrangian of Eq. (3.7) that in the $R$-symmetric limit with $\widetilde{M}_{1}=0$, a decay to a photon cannot occur at tree-level, so that any effects of such operators will be suppressed compared to the others of the same mass dimension.

The values of the Wilson coefficients for the above operators can be found by matching

\footnotetext{
${ }^{9}$ Gauge invariance of the scalar portion of the operator forbids production of goldstone bosons (i.e. longitudinal $W / Z$ bosons), and the heavier higgs bosons $A^{0}, H^{0}$, and $H^{ \pm}$are of course kinematically excluded in the decoupling limit.

${ }^{10}$ We have used integration by parts to move all derivatives off of $\lambda$, and used field redefinitions to eliminate terms proportional to the equations of motion of the goldstino and gauge bosons. We elect not to use field redefinitions to eliminate terms proportional to $\bar{\sigma}^{\mu} \partial_{\mu} \lambda$, as the resulting operators (arising from the gaugino mass term) would violate the $R$-symmetry.

${ }^{11}$ This operator arises from integrating out intermediate sfermions as opposed to higgsinos, so our power counting may be spoiled if there are any relatively light sfermions. We will later explicitly calculate the decay rate for $\lambda \rightarrow \bar{\psi} \psi+\zeta$ at tree level to all orders in $m_{\lambda}^{2} / m_{\phi}^{2}$ to account for this possibility.
} 
onto the original lagrangian of Eq. (3.7):

$$
\begin{aligned}
& C_{R}^{5}=\frac{g^{\prime}\left(\widetilde{m}_{H_{u}}^{2}-\widetilde{m}_{H_{d}}^{2}\right)}{\mu^{2}}, \quad C_{H_{u}, 1}^{6}=\frac{g^{\prime} \widetilde{m}_{H_{u}}^{2}}{\sqrt{2} \mu^{2}}, \quad C_{H_{d}, 1}^{6}=-\frac{g^{\prime} \widetilde{m}_{H_{d}}^{2}}{\sqrt{2} \mu^{2}}, \\
& C_{\psi}^{6}=-\sqrt{2} g^{\prime} Y_{\psi} \frac{\widetilde{m}_{\phi}^{2}}{m_{\phi}^{2}}, \quad C_{H_{u}, 2}^{6}=0, \quad C_{H_{d}, 2}^{6}=0 .
\end{aligned}
$$

Here, $g^{\prime}$ is the hypercharge gauge coupling, $Y_{\psi}$ is the hypercharge of the relevant SM fermion, and the tilded mass parameters are defined in Eq. (3.9). Inverse powers of the higgsino mass-squared $\mu^{2}$ and scalar mass-squared $m_{\phi}^{2}$ appear as expected, since these are the masses of the fields we are integrating out.

The key observation is that the above Wilson coefficients are still order $\mathcal{O}\left(\mu^{0}\right)$ in the higgsino decoupling limit, ${ }^{12}$ since the soft masses scale as $\mathcal{O}\left(\mu^{2}\right)$. Thus, even if the LOSP has negligible higgsino fraction, there are relevant bino-goldstino-higgs couplings. As advertised, the leading decays in the higgsino-decoupling and $R$-symmetric limits are

$$
\lambda \rightarrow h^{0}+\zeta, \quad \lambda \rightarrow Z+\zeta, \quad \lambda \rightarrow \psi \bar{\psi}+\zeta
$$

Now, using the effective operators of Sec. 4.1, we can calculate the various bino LOSP decay widths in the higgsino decoupling and $R$-symmetric limits. Possible $R$-violating decays are described in App. B.

\subsection{Decay to Higgs Bosons}

The contributions to the $\lambda \rightarrow h^{0}+\zeta$ decay from the dimension 5 and dimension 6 operators may be expressed in terms of an effective Yukawa interaction for on-shell states: ${ }^{13}$

$$
\mathcal{L}_{\text {eff }}=-\frac{M_{Z} \mu \sin \theta_{W}}{\sqrt{2} F}\left(C_{\text {net }}^{5}+\frac{m_{\lambda}}{\mu} C_{\text {net }}^{6}\right) \lambda \zeta h^{0} .
$$

The coefficients $C_{\text {net }}^{5}$ and $C_{\text {net }}^{6}$ are appropriate linear combinations of the Wilson coefficients of the dimension 5 and 6 operators, respectively, and are given explicitly in App. B. In the decoupling and $R$-symmetric limits, they take on the values

$$
\begin{aligned}
C_{\text {net }}^{5} & =\frac{\left(\widetilde{m}_{H_{u}}^{2}-\widetilde{m}_{H_{d}}^{2}\right) \sin 2 \beta}{\mu^{2}}, \\
C_{\text {net }}^{6} & =\frac{\widetilde{m}_{H_{u}}^{2} \sin ^{2} \beta-\widetilde{m}_{H_{d}}^{2} \cos ^{2} \beta}{\mu^{2}} .
\end{aligned}
$$

\footnotetext{
${ }^{12}$ Note that they are not of order 1 , but rather of order $\cot \theta$. We have chosen to leave such dependence in the Wilson coefficients, rather than replacing $F$ with $F_{2}$ everywhere, so that the only modification needed to describe the couplings of the eaten goldstino is to remove tildes from all soft mass parameters.

${ }^{13}$ This is not strictly speaking the whole story; the bino may also decay via two local dimension 5 operators $\left(\mathcal{O}_{R}^{5}\right.$ and $\lambda \lambda\left(H_{u} \cdot H_{d}\right)^{*}$ or their wino equivalents) connected by a virtual wino or bino. However, their contributions to the decay amplitude are suppressed by $m_{\lambda} /(\mu \tan \beta)$ compared to that of $O_{R}^{5}$ alone, or $1 / \tan ^{2} \beta$ to those of the dimension 6 operators, and can be safely ignored in most limits.
} 
The decay rate via this channel is

$$
\Gamma=\frac{m_{\lambda} \mu^{2} M_{Z}^{2} \sin ^{2} \theta_{W}}{32 \pi F^{2}}\left(C_{\text {net }}^{5}+\frac{m_{\lambda}}{\mu} C_{\text {net }}^{6}\right)^{2}\left(1-\frac{m_{h^{0}}^{2}}{m_{\lambda}^{2}}\right)^{2}
$$

In the extreme decoupling limit, we would expect the $C_{\text {net }}^{5}$ term, arising from the dimension 5 operator, to dominate over the effects of any dimension 6 operators, which are naturally suppressed by a factor of $m_{\lambda} / \mu$. However, our power counting may be spoiled for large $\tan \beta$, due to the factor of $\sin 2 \beta \approx 2 / \tan \beta$ in $C_{\text {net }}^{5}$. In the event that $\tan \beta$ is of the same order as $\mu / m_{\lambda}$, we cannot neglect the dimension 6 operators. There are no such complications for the dimension 7 or higher operators, which may be safely ignored in the decoupling limit.

As a side note, there are only a few changes to the above calculation if we consider a wino LOSP. There are now two allowed operators at dimension 5 -namely, $\lambda^{a} \zeta\left(H_{u} T^{a} \cdot H_{d}\right)^{*}$ and $\lambda^{a} \zeta\left(H_{u} \cdot T^{a} H_{d}\right)^{*}$ - but the results throughout are almost identical, requiring only the replacement $g^{\prime} \rightarrow-g$ or $\sin \theta_{W} \rightarrow-\cos \theta_{W}$ (as the neutral higgsinos have $T^{3}$ and $Y$ differing only by a sign). In particular, one can verify that there is no net coupling to the $Z$ boson from the dimension 5 operators, ${ }^{14}$ so the neutral wino LOSP decays dominantly to higgs bosons in the small $\left(m_{\lambda} \tan \beta\right) / \mu$ limit.

\subsection{Decay to $Z$ Bosons}

The dimension 5 operator does not contribute to $Z$ decay. The dimension 6 operators mediate the decay $\lambda \rightarrow Z+\zeta$ due to the presence of covariant derivatives. Expanding the lagrangian in unitarity gauge, we find a relatively simple coupling to the $Z$ boson:

$$
\mathcal{L}=\frac{M_{Z}^{2} \sin \theta_{W}}{\sqrt{2} F} C_{\mathrm{net}, Z}^{6} \zeta^{\dagger} \bar{\sigma}^{\mu} \lambda Z_{\mu}
$$

with $C_{\text {net }, Z}^{6}$ being a different linear combination of the Wilson coefficients of the dimension 6 operators. The definition of $C_{\text {net }, Z}^{6}$ is given explicitly in App. B, and attains the value

$$
C_{\mathrm{net}, Z}^{6}=-\frac{\widetilde{m}_{H_{u}}^{2} \sin ^{2} \beta+\widetilde{m}_{H_{d}}^{2} \cos ^{2} \beta}{\mu^{2}}
$$

in the decoupling and $R$-symmetric limit. The resulting decay rate is

$$
\Gamma_{Z}=\frac{M_{Z}^{2} m_{\lambda}^{3} \sin ^{2} \theta_{W}}{32 \pi F^{2}}\left(C_{\text {net }, Z}^{6}\right)^{2}\left(1-\frac{M_{Z}^{2}}{m_{\lambda}^{2}}\right)^{2}\left(1+2 \frac{M_{Z}^{2}}{m_{\lambda}^{2}}\right) .
$$

\footnotetext{
${ }^{14}$ Dimension 5 operators can, however, induce a $\lambda^{ \pm} \rightarrow W^{ \pm} \zeta$ decay. Such decays may well be phenomenologically interesting, as the competing observable-sector decays $\left(\lambda^{ \pm} \rightarrow l^{ \pm} \nu \lambda_{3}, \lambda^{ \pm} \rightarrow \pi^{ \pm} \lambda_{3}\right.$, et al. [23]) can be highly suppressed due to the near-degeneracy of the chargino and wino. However, such decays are certainly not specific to this $R$-symmetric limit, or even to the multiple goldstino model.
} 


\subsection{Decay to Difermions}

Finally, the operator $O_{\psi}^{6}$ mediates the decay of a bino LOSP to a goldstino and a fermion pair. The decay rate from just this operator is

$$
\Gamma_{\psi \bar{\psi}}=\frac{m_{\lambda}^{5} \sec ^{2} \theta_{W}}{32 \pi F^{2}} \frac{\alpha_{\mathrm{EM}} Y_{\psi}^{2}}{12 \pi} \frac{\widetilde{m}_{\phi}^{4}}{m_{\phi}^{4}}
$$

in the limit of vanishing fermion masses. ${ }^{15}$

As argued in Ref. [9], the decay rate is non-zero even in the limit of very large scalar masses. However, due to the factor of $\alpha_{\mathrm{EM}} Y_{\psi}^{2} /(12 \pi)$, the decay rate to fermions is typically subdominant to the higgs and $Z$ modes, even after summing over all possible fermion final states. One might wonder whether there could be an enhancement at moderate values of the scalar masses. Calculating the explicit tree-level decay rate for this mode to all orders in the scalar mass (while still working in the higgsino decoupling limit), the result in Eq. (4.14) is multiplied by a function $f\left[m_{\phi}^{2} / m_{\lambda}^{2}\right]$ :

$$
f[x]=6 x^{2}\left(-5+6 x+2(x-1)(3 x-1) \log \left[1-\frac{1}{x}\right]\right) \simeq 1+\frac{4}{5 x}+\mathcal{O}\left(\frac{1}{x^{2}}\right) .
$$

This function never grows larger than 6 (at $m_{\phi}=m_{\lambda}$ ), and drops off quite sharply from that value as $m_{\phi}$ increases. For example, $m_{\phi}$ must be less than $1.25 m_{\lambda}$ for $f$ to be greater than 2. Thus, the difermion mode is indeed subdominant. The sole exception occurs when $\widetilde{m}_{H_{u}}^{2}$ and $\widetilde{m}_{H_{d}}^{2}$ are both close to zero, where the higgs and $Z$ decay modes are suppressed.

\section{Comparisons to the Gravitino Case}

Before showing results for bino LOSP branching ratios in the next section, it is instructive to compare the $R$-symmetric goldstino results in Sec. 4 to the more familiar case of a gravitino. Indeed, the existence of a bino-goldstino-higgs coupling in the higgsino decoupling limit is quite surprising from the point of view of the more familiar longitudinal gravitino couplings, where it is known that the decay $\lambda \rightarrow h^{0}+\widetilde{G}_{L}$ is highly suppressed. In this section, we will go to the higgsino decoupling limit and calculate the effective interactions for a longitudinal gravitino. In the decoupling limit effective theory, we will find seemingly miraculous cancellations enforced by supercurrent conservation.

\subsection{Additional Operations for the Gravitino}

In the higgsino decoupling limit for a longitudinal gravitino, the operators from Sec. 4.1 persist after the replacement $\zeta \rightarrow \widetilde{G}_{L}$, and they have the same Wilson coefficients as Eq. (4.5) after removing the tildes from the soft mass parameters. In addition, there are

\footnotetext{
${ }^{15}$ We also neglect here possible contributions from interference between diagrams featuring this operator and diagrams in which the fermions originate from an off-shell higgs or $Z$ produced by one of the other dimension 6 operators.
} 
eight $R$-symmetry-violating operators at dimension 5 and 6 which contribute to bino LOSP decay. Their associated Wilson coefficients can again be found by matching. ${ }^{16}$

$$
\begin{aligned}
\mathcal{O}_{\not R, B}^{5} & =C_{\not h, B}^{5} \frac{M_{1}}{F} i \lambda \sigma^{\mu \nu} \widetilde{G}_{L} F_{\mu \nu}, & C_{\not R, B}^{5} & =\frac{1}{\sqrt{2}}, \\
\mathcal{O}_{\not R, H_{u} \cdot H_{d}}^{5} & =C_{\not h, H_{u} \cdot H_{d}}^{5} \frac{\mu}{F} \lambda \widetilde{G}_{L}\left(H_{u} \cdot H_{d}\right), & C_{\not R, H_{u} \cdot H_{d}}^{5} & =0, \\
\mathcal{O}_{\not R, H_{u}}^{5} & =C_{\not R, H_{u}}^{5} \frac{\mu}{F} \lambda \widetilde{G}_{L} H_{u}^{\dagger} H_{u}, & C_{\not R, H_{u}}^{5} & =\frac{g^{\prime}}{\sqrt{2}}\left(\frac{B_{\mu}}{\mu^{2}}-\frac{M_{1}}{2 \mu}\right), \\
\mathcal{O}_{\not R, H_{d}}^{5} & =C_{\not h, d}^{5} \frac{\mu}{F} \lambda \widetilde{G}_{L} H_{d}^{\dagger} H_{d}, & C_{\not R, H_{d}}^{5} & =-\frac{g^{\prime}}{\sqrt{2}}\left(\frac{B_{\mu}}{\mu^{2}}-\frac{M_{1}}{2 \mu}\right), \\
\mathcal{O}_{\not R, 1}^{6} & =\frac{C_{\not R, 1}^{6}}{F} i \widetilde{G}_{L}^{\dagger} \bar{\sigma}^{\mu} \lambda\left(H_{u} \cdot D_{\mu} H_{d}\right)^{*}, & C_{\not R, 1}^{6} & =-\frac{g^{\prime} B_{\mu}}{\sqrt{2} \mu^{2}}, \\
\mathcal{O}_{\not R, 2}^{6} & =\frac{C_{\not R, 2}^{6}}{F} i \widetilde{G}_{L}^{\dagger} \bar{\sigma}^{\mu} \lambda\left(D_{\mu} H_{u} \cdot H_{d}\right)^{*}, & C_{\not R, 2}^{6} & =\frac{g^{\prime} B_{\mu}}{\sqrt{2} \mu^{2}}, \\
\mathcal{O}_{\not R, 3}^{6} & =\frac{C_{\not R, 3}^{6}}{F} i \widetilde{G}_{L}^{\dagger} \bar{\sigma}^{\mu} \lambda\left(H_{u} \cdot D_{\mu} H_{d}\right), & C_{\not R, 3}^{6} & =0, \\
\mathcal{O}_{\not R, 4}^{6} & =\frac{C_{\not R, 4}^{6}}{F} i \widetilde{G}_{L}^{\dagger} \bar{\sigma}^{\mu} \lambda\left(D_{\mu} H_{u} \cdot H_{d}\right), & C_{\not R, 4}^{6} & =0 .
\end{aligned}
$$

The first operator $\mathcal{O}_{B}^{5}$ is exactly the second term in Eq. (3.6). The terms proportional to $M_{1}$ in $C_{\not R, H_{u}}^{5}$ and $C_{\not R, H_{d}}^{5}$ derive from the third term in Eq. (3.6), which contains the auxiliary field $D$. The remaining contributions arise from the $R$-symmetry-violating $B_{\mu}$ term.

Looking at these Wilson coefficients, one might (erroneously) conclude that in the higgsino decoupling limit, a bino LOSP should dominantly decay to a gravitino via a physical higgs instead of via a $\gamma / Z$. After all, the leading order bino-goldstino-higgs couplings come from four dimension-5 operators- $\mathcal{O}_{R}^{5}, \mathcal{O}_{\not h, H_{u} \cdot H_{d}}^{5}, \mathcal{O}_{\not R, H_{u}}^{5}$, and $\mathcal{O}_{\not R, H_{d}}^{5}$-which are enhanced by a factor of $\mu / m_{\lambda}$ compared to the bino-goldstino- $\gamma / Z$ coupling from $\mathcal{O}_{B}^{5}$.

However, we know this not to be the case for the gravitino. From conservation of the supercurrent, the decay rate for $\lambda \rightarrow h^{0}+\widetilde{G}_{L}$ given in Eq. (2.5) is suppressed in the decoupling limit by a factor of $\mathcal{O}\left(m_{\lambda}^{2} M_{Z}^{2} / \mu^{4}\right)$ from the decay rates for $\lambda \rightarrow \gamma / Z+\widetilde{G}_{L}$ given in Eqs. (2.3) and (2.4). Apparently, when calculating the decay rate of a bino LOSP to a higgs boson and a gravitino using the decoupling limit effective field theory, the contributions to the amplitude from the dimension 5 , dimension 6 , and dimension 7 operators yield cancellations up to three orders in the $m_{\lambda} / \mu$ expansion.

\subsection{Miraculous Cancellations}

The easiest way to see that there must be a cancellation is to go back to the gravitino coupling from Eq. (3.6) before integrating out the higgsino. We can make a standard SUSY transformation on all of our visible sector fields with infinitesimal parameter $\widetilde{G}_{L} / F$,

$$
\phi \rightarrow \phi+\frac{1}{F} \psi \widetilde{G}_{L},
$$

\footnotetext{
${ }^{16}$ There are also analogous results in the case of an uneaten goldstino in the absence of an $R$-symmetry, as long as tildes are added to the soft mass parameters and $\widetilde{G}_{L}$ is replaced with $\zeta$. See App. B.
} 
with similar expressions for other fields. This is an allowed field redefinition since it leaves the one-particle states unchanged. Since the coefficients of the SUSY-breaking mass terms and the couplings of $\widetilde{G}_{L}$ are identical up to a sign, the coupling terms (at order $1 / F$ ) cancel under this transformation. This cancellation is special to the eaten goldstino and does not in general occur for an uneaten goldstino. The SUSY-respecting part of the lagrangian will clearly remain unchanged under this field redefinition except for terms proportional to $\partial_{\mu} \widetilde{G}_{L}$. Thus, $\widetilde{G}_{L}$ only couples derivatively to MSSM particles, and does so in exactly the manner described by the supercurrent formalism of Eq. (2.2).

It is also instructive to see how this cancellation works in the decoupling limit effective field theory. The $\lambda \rightarrow h^{0}+\widetilde{G}_{L}$ decay may still be completely parametrized as a Yukawa interaction as in Eq. (4.7) for the leading two orders in $m_{\lambda} / \mu:{ }^{17}$

$$
\mathcal{L}=-\frac{M_{Z} \mu \sin \theta_{W}}{\sqrt{2} F}\left(C_{\text {net }}^{5}+\frac{m_{\lambda}}{\mu} C_{\text {net }}^{6}\right) \lambda \widetilde{G}_{L} h^{0} .
$$

The coefficients $C_{\text {net }}^{5}$ and $C_{\text {net }}^{6}$ have new contributions proportional to $B_{\mu}$ and $M_{1}$ :

$$
\begin{aligned}
C_{\text {net }}^{5} & =\frac{\left(m_{H_{u}}^{2}-m_{H_{d}}^{2}\right) \cos (\alpha+\beta)-2 B_{\mu} \sin (\alpha+\beta)}{\mu^{2}}+\frac{M_{1}}{\mu} \sin (\alpha+\beta), \\
C_{\text {net }}^{6} & =\frac{m_{H_{d}}^{2} \cos \beta \sin \alpha+m_{H_{u}}^{2} \sin \beta \cos \alpha-B_{\mu} \cos (\beta-\alpha)}{\mu^{2}} .
\end{aligned}
$$

If one uses the tree-level relations for the parameters in the higgs potential (see App. A), these simplify considerably:

$$
C_{\mathrm{net}}^{5}=-\frac{M_{1} \cos 2 \beta}{\mu}+\mathcal{O}\left(\frac{M_{Z}^{2}}{\mu^{2}}\right), \quad C_{\mathrm{net}}^{6}=\cos 2 \beta+\mathcal{O}\left(\frac{M_{Z}^{2}}{\mu^{2}}\right) .
$$

We see that the $\mathcal{O}(1)$ term in $C_{\text {net }}^{5}$ have cancelled entirely, and the $\mathcal{O}\left(M_{1} / \mu\right)$ term, which arose from the $\lambda \widetilde{G}_{L} D$ term in Eq. (3.7), cancels against $C_{\text {net }}^{6}$ since $M_{1}=m_{\lambda}$ at this order. Diagrammatically, the first cancellation is among the diagrams in Fig. 5, and the second cancellation is among those in Fig. 7. There is yet another cancellation at the next order in $\mu$ involving dimension 7 operators, but it is not instructive to show it explicitly here; it may be verified using the methods of App. C after diagonalizing the neutralino mass matrix order by order in $\mu$.

\subsection{Why Goldstini are Different}

These miraculous cancellations for the gravitino case, removing the leading three orders of contributions to the bino LOSP decay to higgs, are very specific to the gravitino and the values of its associated Wilson coefficients. There is much more freedom in choosing the couplings of the uneaten goldstino. Concretely, the Wilson coefficients feature the tilded versions of soft SUSY-breaking mass parameters, recalling $\widetilde{M}_{i}=M_{i, 2} \cot \theta-M_{i, 1} \tan \theta$ from Eq. (3.9). These tilded parameters need not satisfy any a priori relation among themselves, and thus the cancellations above will not occur in general for a goldstino.

\footnotetext{
${ }^{17}$ The diagrams featuring two dimension 5 operators connected by a virtual bino or wino cancel separately.
} 

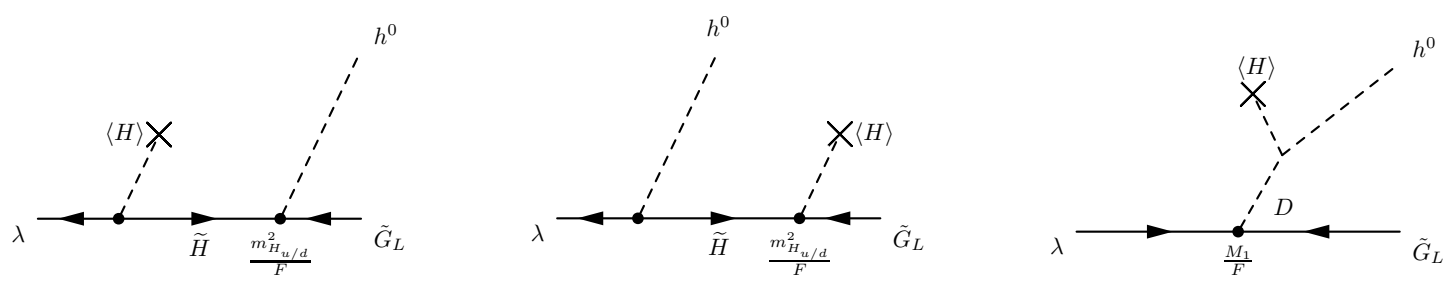

Figure 7: These diagrams, which we would expect to yield $\mathcal{O}\left(\mu^{0}\right)$ contributions to the $\lambda \rightarrow h^{0}+\widetilde{G}_{L}$ amplitude, cancel among themselves.

Said another way, the mechanisms which ensured the cancellations for the gravitino are not applicable in the goldstino case. The field redefinition of Eq. (5.9) made it manifest that the gravitino couples derivatively to observable sector fields, but the same cannot be done in general for the uneaten goldstino. We could attempt to remove one such coupling with the same sort of SUSY transformation, with

$$
\phi \rightarrow \phi+\frac{1}{F} \frac{\widetilde{m}_{\phi}^{2}}{m_{\phi}^{2}} \psi \zeta,
$$

but unless $\widetilde{m}_{\phi}^{2} / m_{\phi}^{2}=\widetilde{M}_{a} / M_{a}$ for all scalar and gaugino mass terms, there is no transformation that will remove all such couplings and make $\zeta$ purely derivatively coupled.

Thus, one expects a variety of counterintuitive LOSP decay patterns in the presence of goldstini, such as wrong-helicity decays like $\tilde{\tau}_{R} \rightarrow \tau_{L}+\zeta$, flavor-violating decays, or reshuffled neutralino/chargino branching fractions. Of course, the phenomenological differences between a longitudinal gravtino and an uneaten goldstino are highlighted when the "standard" decay is forbidden. This is precisely the case for our bino LOSP in the higgsino decoupling and $R$-symmetric limit, where the standard $\gamma / Z$ decay is suppressed and the novel $h^{0}$ mode can dominate.

\section{Branching Ratio Results}

We now discuss the bino LOSP branching ratios in the presence of multiple SUSY breaking sectors, using the $R$-symmetric setup from Fig. 2. In the bulk of parameter space, the decay mode $\lambda \rightarrow \psi \bar{\psi}+\zeta$ is suppressed, so we will first focus on the branching ratios to higgs and $Z$ bosons, neglecting any three-body decays. A brief discussion of what happens away from the $R$-symmetric limit appears in Sec. 6.3.

\subsection{Higgs and $Z$ Boson Branching Ratios}

When three-body decays can be neglected, the dominant phenomenology is determined by the two parameters

$$
\epsilon \equiv \frac{m_{\lambda} \tan \beta}{\mu}, \quad \tan \gamma \equiv \frac{\widetilde{m}_{H_{u}}^{2}}{\widetilde{m}_{H_{d}}^{2}},
$$



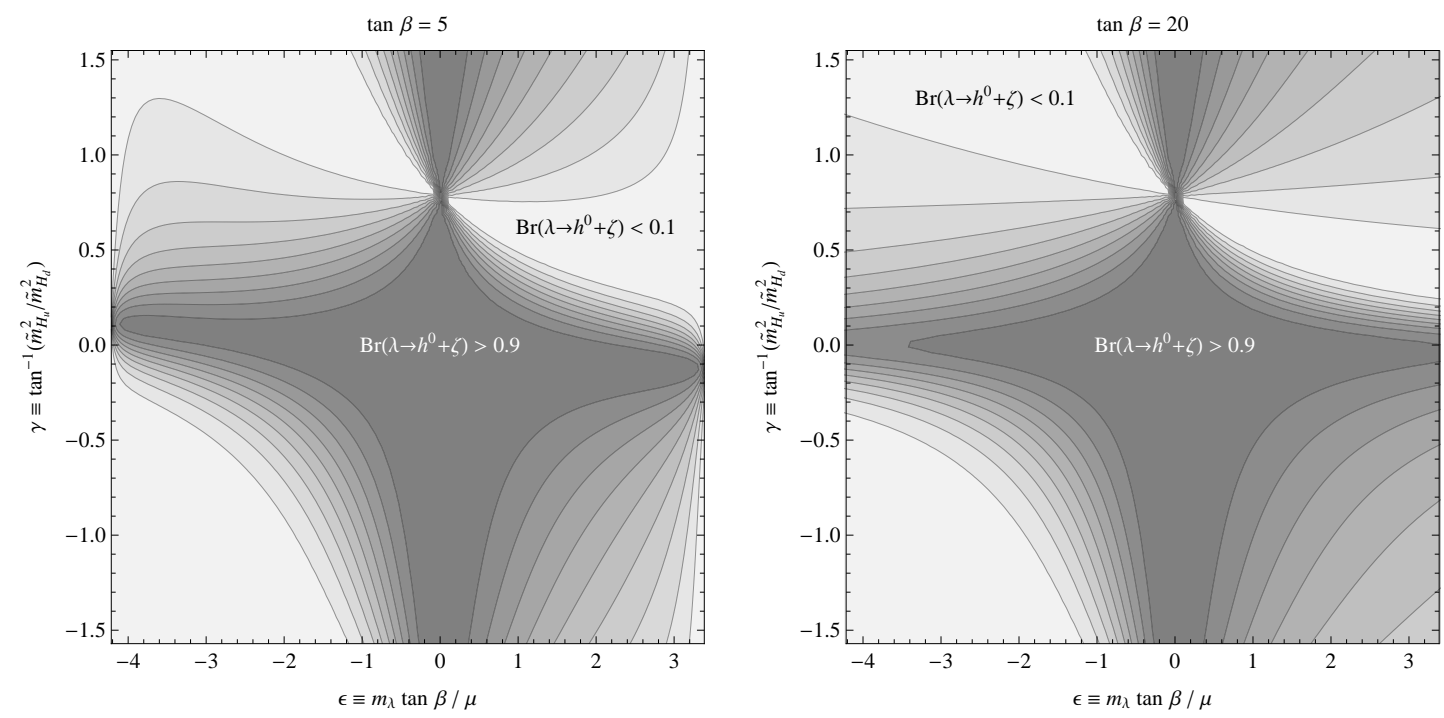

Figure 8: Branching ratios for $\lambda \rightarrow h^{0}+\zeta$ in the $\epsilon^{-} \gamma$ plane for $\tan \beta=5$ (left, same as Fig. 3) and $\tan \beta=20$ (right), respectively. The remaining branching ratio is dominated by $\lambda \rightarrow Z+\zeta$. The main differences between the two plots arise because at larger $\tan \beta$, the kinematically excluded region $m_{\lambda}<m_{h}^{0}$ (which bounds the left plot) is not encountered until larger $\epsilon$. In this and the remaining plots, we have fixed $M_{1}=155 \mathrm{GeV}$ and $m_{h^{0}}=120 \mathrm{GeV}$, which are mainly relevant for setting the phase space factors in the partial widths.

previously mentioned in Eqs. (2.17) and (2.18). Using the partial widths calculated in Eq. (4.10) and Eq. (4.13), the branching ratio for the bino LOSP decay to $h^{0}$ or $Z$, assuming both are kinematically allowed, may be expressed in the relatively compact form:

$$
\operatorname{Br}\left(\lambda \rightarrow h^{0} \zeta\right)=\frac{\left(\frac{\epsilon^{-1}-\epsilon_{0}^{-1}}{\omega}\right)^{2}}{1+\left(\frac{\epsilon^{-1}-\epsilon_{0}^{-1}}{\omega}\right)^{2}}, \quad \operatorname{Br}(\lambda \rightarrow Z \zeta)=\frac{1}{1+\left(\frac{\epsilon^{-1}-\epsilon_{0}^{-1}}{\omega}\right)^{2}} .
$$

In particular, the branching ratio to $Z$ bosons is a Lorentzian in $\epsilon^{-1}$ and is thus negligible for small $\epsilon$, as expected. The Lorentzian is centered at $\epsilon_{0}^{-1}$ with a width $\omega$,

$$
\begin{aligned}
\epsilon_{0}^{-1} & =\frac{1-\tan \gamma \tan ^{2} \beta}{2 \tan ^{2} \beta(\tan \gamma-1)}, \\
\omega & =\frac{1+\tan \gamma \tan ^{2} \beta}{2 \tan ^{2} \beta(\tan \gamma-1)}\left(\frac{m_{\lambda}^{2}-M_{Z}^{2}}{m_{\lambda}^{2}-m_{h^{0}}^{2}} \sqrt{1+2 \frac{M_{Z}^{2}}{m_{\lambda}^{2}}}\right),
\end{aligned}
$$

where the precise values of $\epsilon_{0}^{-1}$ and $\omega$ depend on the higgs soft mass ratio $\tan \gamma, \tan \beta$, and various kinematic factors. Of course, additional three-body decays, whether to fermions or to multiple higgs or $\mathrm{Z}$ bosons, will spoil the simplicity of these expressions.

Plots of the branching ratio to higgs in the $\epsilon^{-\gamma}$ plane are shown in Fig. 8, and slices through that plane are shown in Figs. 9 and 10. In the latter plots, the solid lines are the all-orders tree-level calculations from App. C, while the dashed lines are the analytic 

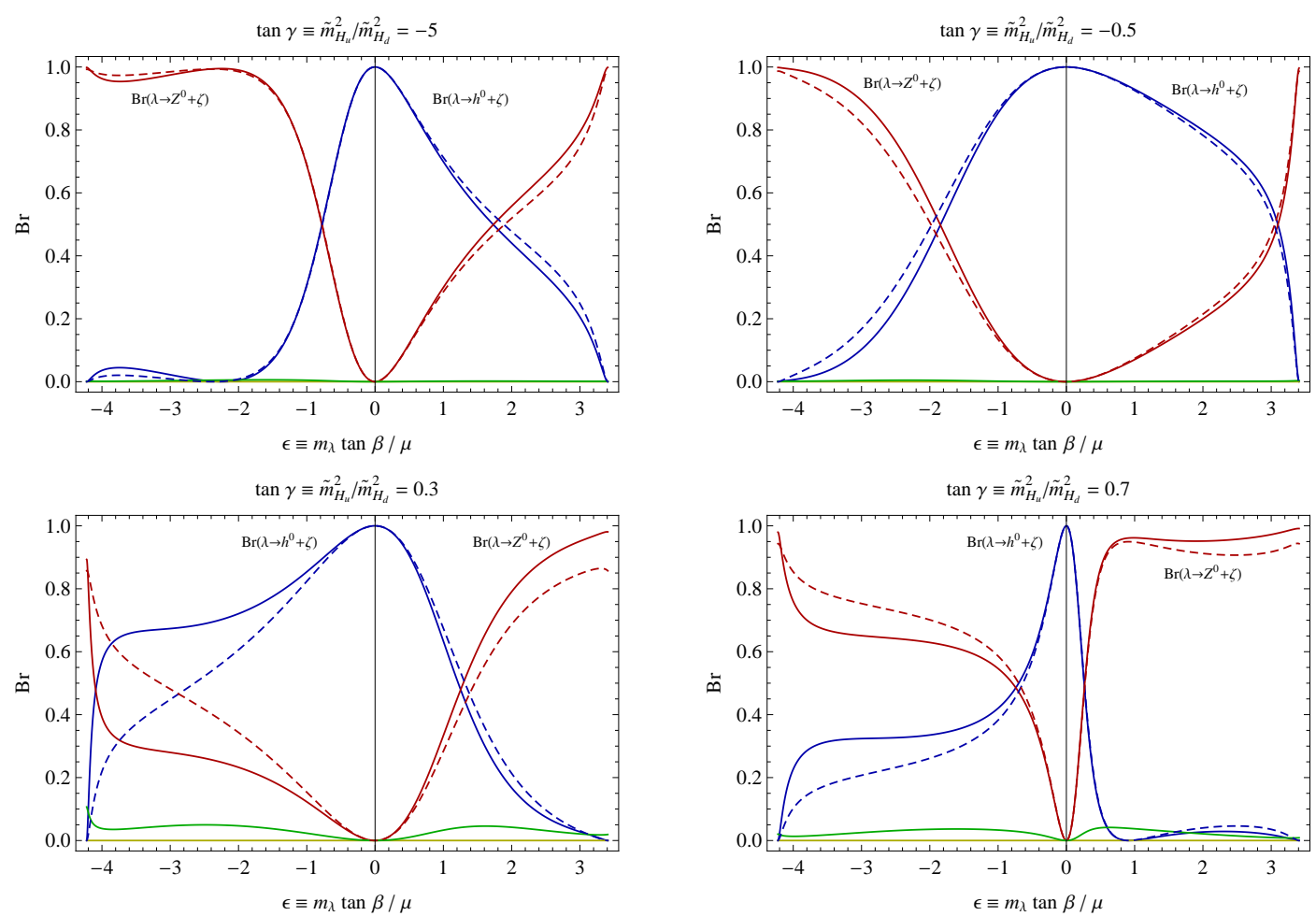

Figure 9: Branching ratios for the bino LOSP as a function of $\epsilon$ for fixed values of $\tan \gamma$. These are all slices of the left plot in Fig. 8 with $\tan \beta=5, M_{1}=155 \mathrm{GeV}$, and $m_{h^{0}}=120 \mathrm{GeV}$. The solid curves are the all-orders result from App. C, while the dashed curves are from the higgsino decoupling effective theory in Sec. 4. The curves are $\operatorname{Br}\left(\lambda \rightarrow h^{0} \zeta\right)$ (blue), $\operatorname{Br}(\lambda \rightarrow Z \zeta)$ (purple), $\operatorname{Br}(\lambda \rightarrow \psi \bar{\psi} \zeta)$ (green), and $\operatorname{Br}(\lambda \rightarrow \gamma \zeta)$ (yellow). The decay to higgses dominates in the small $\epsilon$ limit, with the next most relevant mode being the $Z$. The branching ratio to difermions is calculated using the results of Sec. 6.2, taking the parameter $\rho$ defined in Eq. (6.7) to be 1.0. As advertised, this branching ratio to difermions is very suppressed, and the branching ratio to photons is essentially zero.

results obtained using the higgsino decoupling effective theory from Sec. 4 (while still using the all-orders result for the physical LOSP mass $m_{\lambda}$ ).

The small $\epsilon$ limit corresponds to the extreme higgsino decoupling regime, where not only $|\mu| \gg m_{\lambda}$, but the $\tan \beta$ suppressed dimension 5 operator $\mathcal{O}_{R}^{5}$ dominates over the dimension 6 operators. Thus, generically, for small $\epsilon$, the decay is overwhelmingly to higgs bosons, as expected. However, there is an exception for the region around $\tan \gamma=1$. When $\tan \gamma=1, \widetilde{m}_{H_{u}}^{2}-\widetilde{m}_{H_{d}}^{2}$ and $C_{\text {net }}^{5}$ are both zero and the branching ratios to higgs and $Z$ bosons should be roughly equal up to phase space factors. For tan $\gamma$ slightly removed from unity (downwards for $\epsilon>0$, upwards for $\epsilon<0$ ), $C_{\text {net }}^{5}$ will destructively interfere with $C_{\text {net }}^{6}$ and the $Z$ mode will dominate.

Moving away from small $\epsilon$, we expect the $Z$ branching ratio to increase, as the contributions from dimension 6 operators to bino decay are roughly equal for the higgs and $Z$ modes. This is shown in Fig. 9. The effects of interference between the dimension 5 and dimension 6 operators on the higgs amplitude also grow more pronounced for larger $\epsilon$. For 

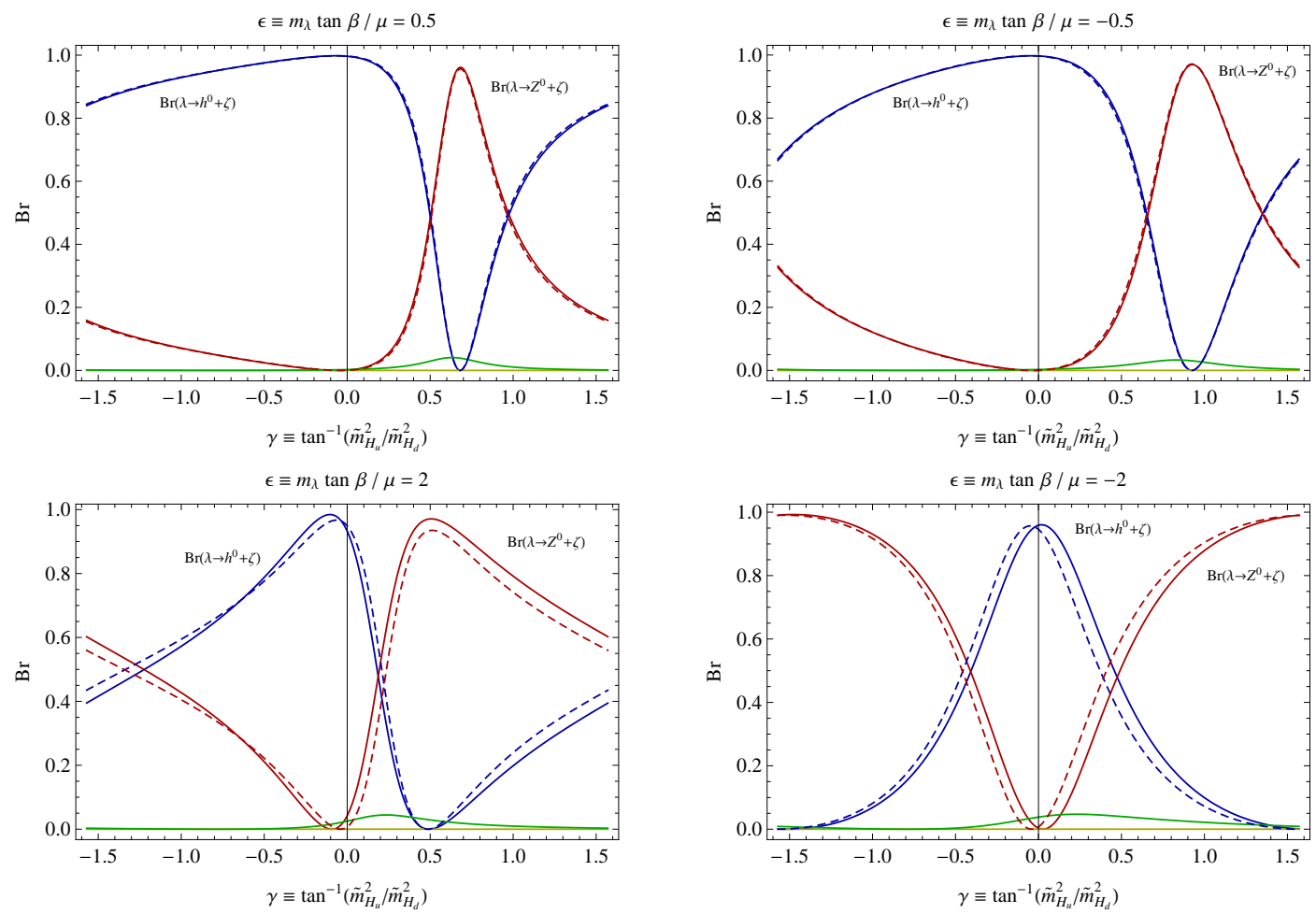

Figure 10: Same as Fig. 9, but with branching ratios given as a function of $\gamma$ for fixed values of $\epsilon$.

$\epsilon>0$, the interference is destructive for $\tan \gamma \in\left(1 / \tan ^{2} \beta, 1\right)$, and vice versa for $\epsilon<0 .{ }^{18}$

For extremely large $\epsilon$, the approximations based on being in the higgsino decoupling limit break down as $m_{\lambda} / \mu$ approaches $\mathcal{O}(1)$. Ultimately, the higgs mode is kinematically excluded once the mass of the lightest neutralino (by now predominantly higgsino) drops below the higgs mass.

\subsection{Difermion Branching Ratio}

In most of parameter space, the decay mode $\lambda \rightarrow \psi \bar{\psi}+\zeta$ is suppressed. We can see this most clearly by comparing the decay rate to all fermion species to the decay rate to a $Z$ :

$$
\frac{\sum_{\psi} \Gamma_{\psi \bar{\psi}}}{\Gamma_{Z}}=\frac{\alpha_{\mathrm{EM}}}{3 \pi \sin ^{2} 2 \theta_{W}} \frac{\sum_{i} Y_{i}^{2} \tau_{i}^{2} f_{i}}{\left(C_{\mathrm{net}, Z}^{6}\right)^{2}} \frac{m_{\lambda}^{2}}{M_{Z}^{2}}\left(1-\frac{M_{Z}^{2}}{m_{\lambda}^{2}}\right)^{-2}\left(1+2 \frac{M_{Z}^{2}}{m_{\lambda}^{2}}\right)^{-1}
$$

where $\tau_{i} \equiv \widetilde{m}_{\phi_{i}}^{2} / m_{\phi_{i}}^{2}$ and $f_{i} \equiv f\left[m_{\phi_{i}}^{2} / m_{\lambda}^{2}\right]$, with the function $f$ defined in Eq. (4.15).

For concreteness, consider the limit where $\tan \beta \gg 1,|\mu|, m_{\phi_{i}} \gg m_{\lambda}$, and the $\tau_{i}$ are all equal to a common value $\tau_{0}$. The sum over SM fermion hypercharges (excluding the presumably kinematically inaccessible top) is 103/12. Assuming that the tree-level relations between the soft masses approximately hold, $C_{\text {net }, Z}^{6}=\tau_{0}$. All the $\tau_{i}$ values then

\footnotetext{
${ }^{18}$ The operative relative sign is that between $\mu$ and $m_{\lambda}$. The $\mathcal{O}^{5}$ operator features an odd power of $\mu$, while the $m_{\lambda}$ factor comes from the $C^{6}$ operators, whose only non-vanishing contributions feature the Dirac equation applied to the external bino spinor.
} 


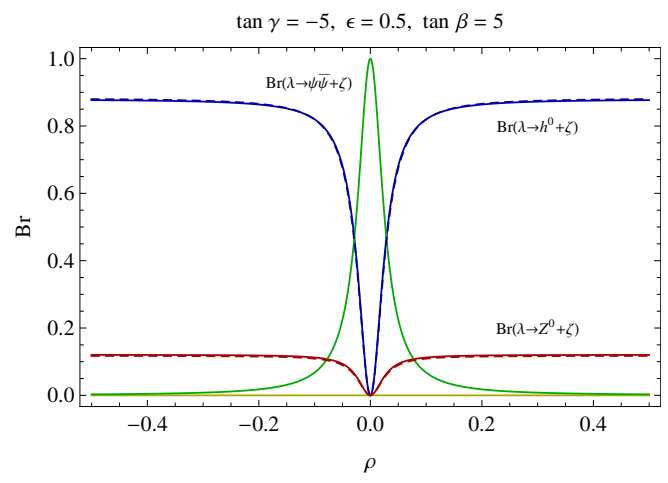

Figure 11: Branching ratios for the bino LOSP as a function of the parameter $\rho$ defined in Eq. (6.7) below, measuring in effect the relative contributions to the higgs and sfermion mass terms by sector 2. If this parameter is tuned close to zero, then the higgs and $Z$ modes shut off, leaving only the difermion channel. For larger values of $\rho$, the difermion channel is suppressed; this occurs generically when the tilded higgs soft mass parameters scale with $\mu^{2}$, as mentioned in Sec. 3.2. For concreteness, all prior figures have used $\rho=1.0$.

cancel, and the net result is

$$
\frac{\sum_{\psi} \Gamma_{\psi \bar{\psi}}}{\Gamma_{Z}} \approx \frac{1}{107} \frac{m_{\lambda}^{2}}{M_{Z}^{2}}\left(1-\frac{M_{Z}^{2}}{m_{\lambda}^{2}}\right)^{-2}\left(1+2 \frac{M_{Z}^{2}}{m_{\lambda}^{2}}\right)^{-1} .
$$

This ratio obtains a minimum of around $1 / 28$ at $m_{\lambda} \approx 140 \mathrm{GeV}$, and it is smaller than $1 / 10$ for $m_{\lambda}$ in the approximate range $100-300 \mathrm{GeV}$.

Of course, there is one somewhat contrived region of parameter space for which the decay to fermions can dominate; if the sector containing the uneaten goldstino gives no contribution to any of the higgs soft masses, then $\widetilde{m}_{H_{u}}^{2}$ and $\widetilde{m}_{H_{d}}^{2}$ vanish and the decay via an off-shell sfermion are the only ones allowed. Fig. 11 shows that the decay to fermions can indeed dominate if the parameter

$$
\rho \equiv \frac{\widetilde{m}_{H_{u}}^{2}+\widetilde{m}_{H_{d}}^{2}}{2 \mu^{2} \cot \theta} \frac{\sum_{i} Y_{i}^{2}}{\sum_{i} Y_{i}^{2} \tau_{i} f_{i}},
$$

with sums taken over all appropriate sfermion species, is tuned close enough to zero.

\subsection{The $R$-violating Regime}

Though not the focus of this paper, we wish to briefly comment on possible $R$-violating decays, for which calculations are given in App. B. As we move away from the $R$-symmetric limit, the LOSP decay to photons is now allowed at tree level, and will generally garner a branching ratio that is at least of the same order as of those to higgs or $Z$. In Fig. 12, we show branching ratios as a function of a parameter $\delta$ which measures the amount of deviation from the $R$-symmetric limit,

$$
\delta \equiv \frac{2}{3} \frac{\tau_{1}+\tau_{2}+\tau_{B_{\mu}}}{\tau_{H_{u}}+\tau_{H_{d}}}
$$




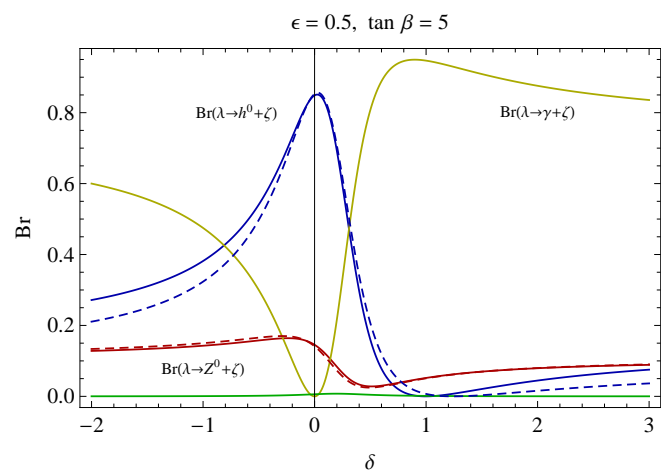

Figure 12: Branching ratios for the bino LOSP as a function of the parameter $\delta$, defined in Eq. (6.8), that measures the deviation from the $R$-symmetric limit. When $\delta=0$, we are in the $R$-symmetric limit of the previous figures. When $\delta=1$, the branching ratios for $\lambda \rightarrow X+\zeta$ are exactly what one would predict for $\lambda \rightarrow X+\widetilde{G}_{L}$ in the more conventional model with only one hidden sector; the photon mode dominates and the higgs mode is highly suppressed.

with $\tau_{i} \equiv \widetilde{M}_{i} / M_{i}$ for any soft mass(-squared) parameter $M_{i}$. In Fig. 12, we hold $\tau_{1}=\tau_{2}=$ $\tau_{B_{\mu}}$ and $\tau_{H_{d}}=\tau_{H_{u}}=\tau_{\phi_{i}}$ for simplicity. When $\delta=0$ we have the exact $R$-symmetric limit; when $\delta=1$ we have the "aligned" limit in which the uneaten goldstino couples simply as a rescaled version of the gravitino (i.e. there is a basis, obtained by making the field redefinition Eq. (5.14), in which it couples only derivatively). Note in the latter limit the higgs branching ratio effectively shuts off, as expected.

The diversity of possible LOSP decay branching ratios shown in Fig. 12 is reminiscent of mixed neutralino LOSP scenarios, where the LOSP has comparable bino, wino, and higgsino fractions. Here, however, we are still working in the higgsino decoupling limit, so the interesting pattern of LOSP widths come not from varying the identity of the LOSP but rather from varying how the hidden sectors couple to the SSM.

\section{Conclusion}

SUSY breaking scenarios with a light gravitino offer fascinating phenomenological possibilities. With the LOSP no longer stable, gravitinos could comprise part or all of the dark matter of the universe, and collider experiments could discover extended SUSY cascade decays. However, the gravitino need not be the only SUSY state lighter than the LOSP. In the context of multiple SUSY breaking, there is a corresponding multiplicity of goldstini whose masses are all typically proportional to $m_{3 / 2}$ (or loop suppressed compared SSM soft masses). Thus, the LOSP may dominantly decay to an uneaten goldstino instead of the gravitino. Since the couplings of the uneaten goldstino are unconstrained by supercurrent conservation, the LOSP can exhibit counterintuitive decay patterns.

In this paper, we have focused on the case of a bino-like LOSP which decays dominantly to higgs bosons despite having negligible higgsino fraction. This effect is particularly pronounced in the presence of a $U(1)_{R}$ symmetry, which suppresses the expected $\lambda \rightarrow \gamma+\zeta$ decay. By studying which low energy effective operators are generated in the higgsino decoupling limit, we have understood why the mode $\lambda \rightarrow h^{0}+\zeta$ dominates in the limit of 
small $\left(m_{\lambda} \tan \beta\right) / \mu$, and also why there is a non-standard $\lambda \rightarrow Z+\zeta$ decay mode away from that limit. We have seen explicitly that there are delicate cancellations in the decay width of the LOSP to a gravitino, and the counterintuitive decays of a LOSP to an uneaten goldstino arise from incomplete cancellations.

Similar counterintuitive decay patterns would be present for a wino-like LOSP, and in general, one should contemplate the possibility of any LOSP decay pattern consistent with SM charges. Those LOSP decays might involve an uneaten goldstino as in this paper, but could also be present with a light axino [24, 25] or a new light hidden sector [26, 27, 28]. To our mind, the most intriguing possibilities involve copious higgs boson production in the final stages of a SUSY cascade decay, which may offer new higgs discovery modes and give further motivation for boosted higgs searches. Studying these phenomena is particularly relevant given the expected LHC sensitivity to SUSY scenarios in the coming two years.

\section{Acknowledgments}

J.T. and Z.T. are supported by the U.S. Department of Energy (D.O.E.) under cooperative research agreement DE-FG02-05ER-41360.

\section{A. Tree-Level Higgs Potential}

The MSSM tree-level higgs potential for the neutral higgs sector arises from a combination of $F$-terms, $D$-terms, and three soft SUSY-breaking terms:

$$
\begin{gathered}
V\left(H_{u}^{0}, H_{d}^{0}\right)=\left(|\mu|^{2}+m_{H_{u}}^{2}\right)\left|H_{u}^{0}\right|^{2}+\left(|\mu|^{2}+m_{H_{d}}^{2}\right)\left|H_{d}^{0}\right|^{2}+B_{\mu}\left(H_{u}^{0} H_{d}^{0}+H_{u}^{0 *} H_{d}^{0 *}\right) \\
+\frac{g^{2}+g^{\prime 2}}{8}\left(\left|H_{u}^{0}\right|^{2}-\left|H_{d}^{0}\right|^{2}\right)^{2}
\end{gathered}
$$

Once we recall that

$$
\begin{aligned}
M_{Z}^{2} & =\frac{1}{2}\left(g^{2}+g^{\prime 2}\right)\left(\left\langle H_{u}^{0}\right\rangle^{2}+\left\langle H_{d}^{0}\right\rangle^{2}\right), \\
\tan \beta & \equiv\left\langle H_{u}^{0}\right\rangle /\left\langle H_{d}^{0}\right\rangle
\end{aligned}
$$

we can use the fact that the vacuum must minimize the higgs potential to find relations among these parameters.

$$
\begin{aligned}
& 0=m_{H_{u}}^{2}+|\mu|^{2}+B_{\mu} \cot \beta-M_{Z}^{2} \frac{\cos 2 \beta}{2}, \\
& 0=m_{H_{d}}^{2}+|\mu|^{2}+B_{\mu} \tan \beta+M_{Z}^{2} \frac{\cos 2 \beta}{2} .
\end{aligned}
$$

It is convenient to take linear combinations of these relations, one without $|\mu|^{2}$ and one without $B_{\mu}$ :

$$
\begin{aligned}
& 0=\left(m_{H_{u}}^{2}-m_{H_{d}}^{2}\right) \sin 2 \beta+2 B_{\mu} \cos 2 \beta-M_{Z}^{2} \frac{\sin 4 \beta}{2}, \\
& 0=m_{H_{u}}^{2} \sin ^{2} \beta-m_{H_{d}}^{2} \cos ^{2} \beta-|\mu|^{2} \cos 2 \beta-M_{Z}^{2} \frac{\cos 2 \beta}{2} .
\end{aligned}
$$


In the higgsino decoupling limit $\left(|\mu|^{2}, m_{A^{0}}^{2} \gg M_{Z}^{2}\right)$, we may neglect the terms proportional to $M_{Z}^{2}$. Also in the same limit, the tree-level relation for the physical higgs mixing angle $\alpha$ simplifies considerably:

$$
\tan 2 \alpha=\tan 2 \beta \frac{m_{A^{0}}^{2}+M_{Z}^{2}}{m_{A^{0}}^{2}-M_{Z}^{2}} \Rightarrow \alpha=\beta-\pi / 2+\mathcal{O}\left(\frac{M_{Z}^{2}}{m_{A^{0}}^{2}}\right) .
$$

Once one applies Eq. (A.8), the relations Eqs. (A.6) and (A.7) are precisely those which cause the cancellation of the $\lambda \rightarrow h^{0}+\widetilde{G}_{L}$ amplitude at the first two orders in $\mu / M_{1}$ in Eqs. (5.11) and (5.12). Another linear combination of Eqs. (A.4) and (A.5) gives a (non-independent) relationship that can be useful for simplifying $C_{\text {net, } Z}^{6}$,

$$
0=|\mu|^{2}+m_{H_{u}}^{2} \sin ^{2} \beta+m_{H_{d}}^{2} \cos ^{2} \beta+B_{\mu} \sin 2 \beta+M_{Z}^{2} \frac{\cos ^{2} 2 \beta}{2} .
$$

A third relation, involving the pseudoscalar mass $m_{A^{0}}^{2}$, allows us to solve for all three soft mass parameters if desired:

$$
\begin{aligned}
B_{\mu} & =-\frac{1}{2} m_{A^{0}}^{2} \sin 2 \beta, \\
m_{H_{u}}^{2} & =-|\mu|^{2}+m_{A^{0}}^{2} \cos ^{2} \beta+M_{Z}^{2} \frac{\cos 2 \beta}{2}, \\
m_{H_{d}}^{2} & =-|\mu|^{2}+m_{A^{0}}^{2} \sin ^{2} \beta-M_{Z}^{2} \frac{\cos 2 \beta}{2} .
\end{aligned}
$$

Of course, all of the above relations are valid only at tree-level, and one does expect corrections to these relations from the same loop effects needed to raise the physical higgs mass above the LEP bounds.

\section{B. $R$-Symmetry Violating Decays}

In the body of this paper, we focused on the setup in Fig. 2 where sector 2 preserves an $R$-symmetry. If the sector 2 does not preserve an $R$-symmetry, then there are many more allowed operators that can mediate the decay of a bino LOSP to the uneaten goldstino. They are exactly those previously given for the gravitino in Sec. 5.1, except with the replacement of $\widetilde{G}_{L}$ with $\zeta$ and with all soft masses tilded.

A decay to photon at tree-level is now allowed through the usual operator

$$
\mathcal{O}_{\not h, B}^{5}=\frac{i \widetilde{M}_{1}}{\sqrt{2} F} \lambda \sigma^{\mu \nu} \zeta F_{\mu \nu},
$$

with resultant decay rate

$$
\Gamma_{\gamma}=\frac{\widetilde{M}_{1}^{2} m_{\lambda}^{3} \cos ^{2} \theta_{W}}{16 \pi F^{2}} .
$$

The couplings of the bino LOSP to the physical higgs $h^{0}$ and any further couplings to the $Z$ not already found in $\mathcal{O}_{R, B}^{5}$ may be parametrized at the first two orders in $m_{\lambda} / \mu$ as

$$
\mathcal{L}=-\frac{M_{Z} \mu \sin \theta_{W}}{\sqrt{2} F}\left[\left(C_{\text {net }}^{5}+\frac{m_{\lambda}}{\mu} C_{\text {net }}^{6}\right) \lambda \zeta h^{0}-\frac{M_{Z}}{\mu} C_{\text {net }, Z}^{6} \zeta^{\dagger} \bar{\sigma}^{\mu} \lambda Z_{\mu}\right]
$$


with $C_{\text {net }}$ representing the following linear combinations of Wilson coefficients:

$$
\begin{aligned}
\frac{g^{\prime}}{\sqrt{2}} C_{\mathrm{net}}^{5}= & \left(C_{R}^{5}+C_{\not h, H_{u} \cdot H_{d}}^{5}\right) \cos (\alpha+\beta) \\
& -2 C_{\not R, H_{u}}^{5} \sin \beta \cos \alpha+2 C_{\not R, H_{d}}^{5} \cos \beta \sin \alpha, \\
\frac{g^{\prime}}{\sqrt{2}} C_{\mathrm{net}}^{6}= & \left(C_{H_{u}, 1}^{6}+C_{H_{u}, 2}^{6}\right) \sin \beta \cos \alpha-\left(C_{H_{d}, 1}^{6}+C_{H_{d}, 2}^{6}\right) \cos \beta \sin \alpha \\
& +\left(C_{\not R, 1}^{6}+C_{\not R, 3}^{6}\right) \sin \beta \sin \alpha-\left(C_{\not R, 2}^{6}+C_{\not R, 4}^{6}\right) \cos \beta \cos \alpha, \\
\frac{g^{\prime}}{\sqrt{2}} C_{\mathrm{net}, Z}^{6}=- & \left(C_{H_{u}, 1}^{6}-C_{H_{u}, 2}^{6}\right) \sin ^{2} \beta+\left(C_{H_{d}, 1}^{6}-C_{H_{d}, 2}^{6}\right) \cos ^{2} \beta \\
& +\frac{1}{2}\left(C_{\not R, 1}^{6}-C_{\not R, 2}^{6}-C_{\not R, 3}^{6}+C_{\not h, 4}^{6}\right) \sin 2 \beta .
\end{aligned}
$$

Here, the factors of $g^{\prime} / \sqrt{2}$ are inserted purely for convenience. In the $R$-symmetric limit, all the $C_{\not R}$ are of course zero.

For the decay to higgs, the formula Eq. (4.10) for the decay rate still holds, but $C_{\text {net }}^{5}$ now has contributions proportional to $\widetilde{B}_{\mu}$ and $\widetilde{M}_{1}$, as it did in Eq. (5.11):19

$$
C_{\mathrm{net}}^{5}=\frac{\left(\widetilde{m}_{H_{u}}^{2}-\widetilde{m}_{H_{d}}^{2}\right) \sin 2 \beta+2 \widetilde{B}_{\mu} \cos 2 \beta}{\mu^{2}}-\frac{\widetilde{M}_{1}}{\mu} \cos 2 \beta .
$$

For the decay to $Z, C_{\text {net, } Z}^{6}$ obtains a term proportional to $\widetilde{B}_{\mu}$

$$
C_{\mathrm{net}, Z}^{6}=-\frac{\widetilde{m}_{H_{u}}^{2} \sin ^{2} \beta+\widetilde{m}_{H_{d}}^{2} \cos ^{2} \beta+\widetilde{B}_{\mu} \sin 2 \beta}{\mu^{2}},
$$

and we must also include the effects of $\widetilde{M}_{1}$ from $\mathcal{O}_{\not R, B}^{5}$ to find the full decay rate:

$$
\begin{aligned}
\Gamma_{Z}= & \frac{m_{\lambda}^{3} \widetilde{M}_{1}^{2} \sin ^{2} \theta_{W}}{16 \pi F^{2}}\left(1-\frac{M_{Z}^{2}}{m_{\lambda}^{2}}\right)^{2} \\
& \times\left(1+\frac{1}{2} \frac{M_{Z}^{2}}{m_{\lambda}^{2}}-\frac{3 M_{Z}^{2} C_{\mathrm{net}, Z}^{6}}{\widetilde{M}_{1} m_{\lambda}}+\left(\frac{M_{Z} C_{\mathrm{net}, Z}^{6}}{\sqrt{2} \widetilde{M}_{1}}\right)^{2}\left(1+2 \frac{M_{Z}^{2}}{m_{\lambda}^{2}}\right)\right) .
\end{aligned}
$$

For the gravitino, $C_{\text {net }, Z}^{6}$ simplifies to unity at this order due to the tree-order relation Eq. (A.9), and the complicated expression in Eq. (B.9) simplifies to the same result we obtained from the supercurrent in Eq. (2.4), as it must. We demonstrated in Sec. 5 that the decay rate to higgs bosons simplifies similarly and in fact completely cancels at this order. For an uneaten goldstino, however, such cancellations do not generically occur, unless the ratio $\tau_{i} \equiv \widetilde{M}_{i} / M_{i}$ is equal for all soft SUSY-breaking mass(-squared) terms $M_{i}$. It is precisely when all the $\tau_{i}$ are equal that one can make the field redefinition Eq. (5.14) to make the goldstino couple only derivatively to visible-sector fields. In this limit, it would

\footnotetext{
${ }^{19}$ Again, we use the approximation $\alpha \approx \beta-\pi / 2$ from Eq. (A.8), which is appropriate at this order in $m_{\lambda} / \mu$. This eliminates a term proportional to $\widetilde{B}_{\mu} \cos (\beta-\alpha)$ in $C_{\text {net }}^{6}$.
} 
couple in exactly the same way as the longitudinal gravitino, except with an enhancement factor of $\tau^{2} \sim \cot ^{2} \theta$. Of course, we should not expect such alignment to occur in general (if only due to loop corrections), so a generic uneaten goldstino will have branching ratios to photons, $Z$ s, and higgses of roughly the same order of magnitude, as suggested by Fig. 12 .

\section{All-Orders Tree-Level Calculation}

The higgsino decoupling limit studied in Sec. 4 is convenient for understanding the physical origin of the counterintuitive LOSP decays, but it is tedious in practice for moderate values of $\mu$. Instead of integrating out the higgsinos and finding an arbitrarily long series of operators and associated Wilson coefficients, we may conduct the calculation with the original lagrangian in the mass eigenstate basis. As long as one can explicitly diagonalize the $4 \times 4$ neutralino mass matrix (analytically or numerically), one can perform the full tree-level calculation to all orders in $\mu$.

To do so, we parametrize the relevant interactions from Eq. (3.5) as follows:

$$
\begin{aligned}
\mathcal{L}=- & \frac{1}{2} M_{i j} \chi_{i} \chi_{j}+\rho_{i} \zeta \chi_{i}-\frac{1}{2} Y_{i j} \chi_{i} \chi_{j} h^{0}+y_{i} \zeta \chi_{i} h^{0} \\
& +G_{i j} \chi_{i}^{\dagger} \bar{\sigma}^{\mu} \chi_{j} Z_{\mu}+L_{i} i \zeta \sigma^{\mu \nu} \chi_{i} \partial_{\mu} Z_{\nu} .
\end{aligned}
$$

In the $\left\{\lambda_{B}, \lambda_{3}, \widetilde{H}_{d}^{0}, \widetilde{H}_{u}^{0}\right\}$ basis, the neutralino mass matrix is [29]

$$
M=\left(\begin{array}{cccc}
M_{1} & 0 & -M_{Z} c_{\beta} s_{W} & M_{Z} s_{\beta} s_{W} \\
0 & M_{2} & M_{Z} c_{\beta} c_{W} & -M_{Z} s_{\beta} c_{W} \\
-M_{Z} c_{\beta} s_{W} & M_{Z} c_{\beta} c_{W} & 0 & -\mu \\
M_{Z} s_{\beta} s_{W} & -M_{Z} s_{\beta} c_{W} & -\mu & 0
\end{array}\right)
$$

the linear mixing with the uneaten goldstino is

$$
\rho=\frac{v}{\sqrt{2} F}\left(\begin{array}{c}
\frac{1}{4} g^{\prime} v \widetilde{M}_{1} \cos 2 \beta \\
-\frac{1}{4} g v \widetilde{M}_{2} \cos 2 \beta \\
\widetilde{m}_{H_{d}}^{2} c_{\beta}+\widetilde{B}_{\mu} s_{\beta} \\
\widetilde{m}_{H_{u}}^{2} s_{\beta}+\widetilde{B}_{\mu} c_{\beta}
\end{array}\right)
$$

the couplings to the physical higgs boson are

$$
Y=\frac{1}{2}\left(\begin{array}{cccc}
0 & 0 & g^{\prime} s_{\alpha} & g^{\prime} c_{\alpha} \\
0 & 0 & -g s_{\alpha} & -g c_{\alpha} \\
g^{\prime} s_{\alpha}-g s_{\alpha} & 0 & 0 \\
g^{\prime} c_{\alpha}-g c_{\alpha} & 0 & 0
\end{array}\right), \quad y=\frac{1}{\sqrt{2} F}\left(\begin{array}{c}
-\widetilde{M}_{1} M_{Z} s_{W} \sin (\alpha+\beta) \\
\widetilde{M}_{2} M_{Z} c_{W} \sin (\alpha+\beta) \\
\widetilde{B}_{\mu} c_{\alpha}-\widetilde{m}_{H_{d}}^{2} s_{\alpha} \\
\widetilde{m}_{H_{u}}^{2} c_{\alpha}-\widetilde{B}_{\mu} s_{\alpha}
\end{array}\right)
$$

and the couplings to the $Z$ boson are

$$
G=\frac{g}{2 c_{W}}\left(\begin{array}{cccc}
0 & 0 & 0 & 0 \\
0 & 0 & 0 & 0 \\
0 & 0 & 1 & 0 \\
0 & 0 & 0 & -1
\end{array}\right), \quad L=\frac{\sqrt{2}}{F}\left(\begin{array}{c}
\widetilde{M}_{1} s_{W} \\
-\widetilde{M}_{2} c_{W} \\
0 \\
0
\end{array}\right)
$$


In the above matrixes, we have used the notation $c_{\theta} \equiv \cos \theta$ and $s_{\theta} \equiv \sin \theta$, with $W$ standing for the weak mixing angle $\theta_{W}$.

To calculate the decays of the lightest neutralino, we go to the mass eigenstate basis:

$$
M \rightarrow M^{\prime}=P^{T} M P
$$

with $P$ chosen to make $M^{\prime}$ diagonal. Note that we treat the linear mixing with the uneaten goldstino as an insertion, which is valid to leading order in $1 / F$. The other matrices and vectors rotate as

$$
\rho \rightarrow \rho^{\prime}=P^{T} \rho, \quad Y \rightarrow Y^{\prime}=P^{T} Y P,
$$

and so forth. The full tree-level amplitude for the decay of the lightest neutralino to a higgs/ $Z$ and a goldstino is thus:

$$
\begin{aligned}
\Gamma_{h^{0}} & =\frac{m_{\lambda}}{16 \pi}\left(y_{1}^{\prime}-\sum_{i} \frac{Y_{1 i}^{\prime} \rho_{i}^{\prime}}{m_{\chi_{i}^{0}}}\right)^{2}\left(1-\frac{m_{h^{0}}^{2}}{m_{\lambda}^{2}}\right)^{2}, \\
\Gamma_{Z} & =\frac{m_{\lambda}^{3}}{16 \pi}\left(1-\frac{M_{Z}^{2}}{m_{\lambda}^{2}}\right)^{2}\left(\frac{L_{1}^{\prime 2}}{2}\left(1+\frac{1}{2} \frac{M_{Z}^{2}}{m_{\lambda}^{2}}\right)-3 \frac{L_{1}^{\prime} K^{\prime}}{m_{\lambda}}+\frac{K^{\prime 2}}{M_{Z}^{2}}\left(1+2 \frac{M_{Z}^{2}}{m_{\lambda}^{2}}\right)\right),
\end{aligned}
$$

where the neutralino masses are labeled by $m_{\chi_{i}^{0}}$, the LOSP mass is $m_{\lambda} \equiv m_{\chi_{1}^{0}}$, and

$$
K^{\prime} \equiv \sum_{i} \frac{G_{1 i}^{\prime} \rho_{i}^{\prime}}{m_{\chi_{i}^{0}}}
$$

A similar calculation for difermion production is beyond the scope of this work; it would in general need to include the effects of $A$-terms, finite fermion masses, and sfermion mixing for the third generation, as well as possible interference from difermions produced by off-shell $Z$ bosons.

\section{References}

[1] S. Dimopoulos, M. Dine, S. Raby, and S. D. Thomas, Experimental Signatures of Low Energy Gauge Mediated Supersymmetry Breaking, Phys. Rev. Lett. 76 (1996) 3494-3497, [hep-ph/9601367].

[2] S. Ambrosanio, G. L. Kane, G. D. Kribs, S. P. Martin, and S. Mrenna, Supersymmetric analysis and predictions based on the CDF eer $\gamma+$ missing $E_{T}$ event, Phys. Rev. Lett. 76 (1996) 3498-3501, [hep-ph/9602239].

[3] S. Dimopoulos, S. D. Thomas, and J. D. Wells, Implications of low energy supersymmetry breaking at the Tevatron, Phys. Rev. D54 (1996) 3283-3288, [hep-ph/9604452].

[4] S. Ambrosanio, G. L. Kane, G. D. Kribs, S. P. Martin, and S. Mrenna, Search for supersymmetry with a light gravitino at the Fermilab Tevatron and CERN LEP colliders, Phys. Rev. D54 (1996) 5395-5411, [hep-ph/9605398].

[5] P. Fayet, Mixing Between Gravitational and Weak Interactions Through the Massive Gravitino, Phys. Lett. B70 (1977) 461. 
[6] P. Fayet, Scattering Cross-Sections of the Photino and the Goldstino (Gravitino) on Matter, Phys. Lett. B86 (1979) 272.

[7] R. Casalbuoni, S. De Curtis, D. Dominici, F. Feruglio, and R. Gatto, A GRAVITINO GOLDSTINO HIGH-ENERGY EQUIVALENCE THEOREM, Phys. Lett. B215 (1988) 313.

[8] R. Casalbuoni, S. De Curtis, D. Dominici, F. Feruglio, and R. Gatto, High-Energy Equivalence Theorem in Spontaneously Broken Supergravity, Phys. Rev. D39 (1989) 2281.

[9] C. Cheung, Y. Nomura, and J. Thaler, Goldstini, JHEP 03 (2010) 073, [arXiv:1002.1967].

[10] N. Craig, J. March-Russell, and M. McCullough, The Goldstini Variations, JHEP 10 (2010) 095, [arXiv: 1007.1239].

[11] R. Argurio, Z. Komargodski, and A. Mariotti, Pseudo-Goldstini in Field Theory, arXiv:1102.2386.

[12] C. Cheung, J. Mardon, Y. Nomura, and J. Thaler, A Definitive Signal of Multiple Supersymmetry Breaking, JHEP 07 (2010) 035, [arXiv: 1004.4637].

[13] H.-C. Cheng, W.-C. Huang, I. Low, and A. Menon, Goldstini as the decaying dark matter, arXiv: 1012.5300

[14] G. D. Kribs, E. Poppitz, and N. Weiner, Flavor in supersymmetry with an extended R-symmetry, Phys. Rev. D78 (2008) 055010, [arXiv:0712.2039].

[15] S. D. L. Amigo, A. E. Blechman, P. J. Fox, and E. Poppitz, R-symmetric gauge mediation, JHEP 01 (2009) 018, [arXiv:0809.1112].

[16] J. M. Butterworth, A. R. Davison, M. Rubin, and G. P. Salam, Jet substructure as a new Higgs search channel at the LHC, Phys. Rev. Lett. 100 (2008) 242001, [arXiv:0802.2470].

[17] G. D. Kribs, A. Martin, T. S. Roy, and M. Spannowsky, Discovering the Higgs Boson in New Physics Events using Jet Substructure, Phys. Rev. D81 (2010) 111501, [arXiv:0912.4731].

[18] A. Abdesselam et al., Boosted objects: a probe of beyond the Standard Model physics, arXiv: 1012.5412.

[19] H. K. Dreiner, H. E. Haber, and S. P. Martin, Two-component spinor techniques and Feynman rules for quantum field theory and supersymmetry, Phys. Rept. 494 (2010) 1-196, [arXiv:0812.1594].

[20] P. Meade, M. Reece, and D. Shih, Prompt Decays of General Neutralino NLSPs at the Tevatron, JHEP 05 (2010) 105, [arXiv:0911.4130].

[21] Z. Komargodski and N. Seiberg, From Linear SUSY to Constrained Superfields, JHEP 09 (2009) 066, [arXiv:0907.2441].

[22] P. Binetruy, G. Girardi, and R. Grimm, Supergravity couplings: a geometric formulation, Phys. Rept. 343 (2001) 255-462, [hep-th/0005225].

[23] C. H. Chen, M. Drees, and J. F. Gunion, A non-standard string/SUSY scenario and its phenomenological implications, Phys. Rev. D55 (1997) 330-347, [hep-ph/9607421].

[24] J. E. Kim, A COMMON SCALE FOR THE INVISIBLE AXION, LOCAL SUSY GUTs AND SAXINO DECAY, Phys. Lett. B136 (1984) 378.

[25] K. Rajagopal, M. S. Turner, and F. Wilczek, Cosmological implications of axinos, Nucl. Phys. B358 (1991) 447-470. 
[26] N. Arkani-Hamed and N. Weiner, LHC Signals for a SuperUnified Theory of Dark Matter, JHEP 12 (2008) 104, [arXiv: 0810.0714].

[27] A. De Simone, M. Garny, A. Ibarra, and C. Weniger, Supersymmetric Leptogenesis with a Light Hidden Sector, JCAP 1007 (2010) 017, [arXiv: 1004.4890].

[28] C. Cheung and Y. Nomura, Singlet Portal to the Hidden Sector, JHEP 11 (2010) 103, [arXiv: 1008.5153].

[29] S. P. Martin, A Supersymmetry Primer, hep-ph/9709356. 